\title{
Quantum damping of skyrmion crystal eigenmodes due to spontaneous quasiparticle decay
}

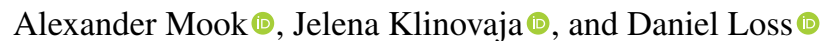 \\ Department of Physics, University of Basel, Klingelbergstrasse 82, 4056 Basel, Switzerland
}

(Received 2 March 2020; revised 4 June 2020; accepted 29 July 2020; published 25 September 2020)

\begin{abstract}
Skyrmion crystals support chiral magnonic edge states akin to electronic quantum Hall edge states. However, magnonic topology relies on the harmonic approximation, neglecting ubiquitous magnon-magnon interactions that yield a finite zero-temperature quantum damping. We demonstrate that spontaneous quasiparticle decay in two-dimensional ferromagnetic skyrmion crystals is a delicate issue, with the quantum damping ranging over several orders of magnitude. Flat magnon bands cause exceptionally strong spontaneous decay at twice their energy. The resulting externally controllable energy-selective magnon breakdown is measurable not only by scattering but also by magnetic resonance experiments, probing the magnetically active anticlockwise, breathing, and clockwise modes. They exhibit distinct decay behavior, with the clockwise (anticlockwise) mode being the least (most) stable mode out of the three. The quantum damping of the topologically nontrivial anticlockwise mode is negligible, establishing the harmonic theory as a trustworthy approximation at low energies, implying excellent prospects of topological magnonics in skyrmion crystals.
\end{abstract}

DOI: 10.1103/PhysRevResearch.2.033491

\section{INTRODUCTION}

Magnetic skyrmion crystals (SkXs), as depicted in Fig. 1, are attracting much attention. These arrays of topologically nontrivial magnetic whirls appear in bulk, multilayers, and thin films, at elevated and zero temperature, in metals and insulators [1-18]. Besides numerous fundamental discoveries [19-28], they have spawned visions of future spintronic [29-31], logic [32-37], quantum information [38-40], and magnonic applications [41-53]. The three magnetically active SkX eigenmodes, associated with an anticlockwise, breathing, or clockwise motion of the skyrmion core [23], belong to the three lowest topologically nontrivial magnon bands [44,48,52], supporting chiral edge magnons [54-56], akin to electronic quantum Hall edge states. Since these chiral edge magnons are innately free of Joule heating, immune to backscattering, and robust against considerable disorder, they are proposed as "information highways" in the field of topological magnonics [57]. However, such fundamental physics reveals itself only at low temperatures, necessitating pushing the frontiers of skyrmionics to the ultraquantum regime, a trend that began only recently [58-72]. Importantly, even at zero temperature, interactions between magnons are not frozen out and spontaneous quasiparticle decay (SQD) [73-110], as sketched in Fig. 1, dresses magnons with intrinsic lifetimes, potentially spoiling the concept of topological magnonics, which relies on the harmonic approximation.

Published by the American Physical Society under the terms of the Creative Commons Attribution 4.0 International license. Further distribution of this work must maintain attribution to the author(s) and the published article's title, journal citation, and DOI.
Herein we explore the many-body quantum physics of magnons in two-dimensional ferromagnetic SkXs and establish them as materials of extremes. Some magnon bands are flat akin to Landau levels, giving rise to sharp peaks in the density of states (DOS) of the two-magnon continuum, into which single magnons decay. Manipulating these peaks by a magnetic field allows for a field-tunable energy-selective magnon breakdown detectable by scattering or absorption experiments. We reveal that the anticlockwise (clockwise) mode is the most (least) stable out of the three magnetically active modes. Even for ultrasmall skyrmions, the damping of the topologically nontrivial anticlockwise mode is negligible, establishing excellent prospects of low-energy topological magnonics in SkXs.

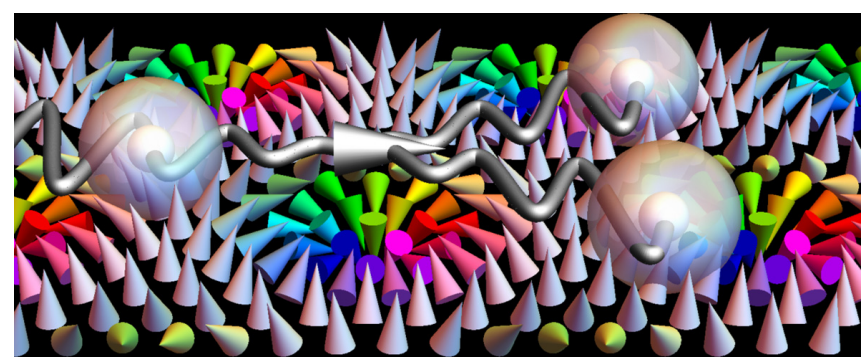

FIG. 1. Sketch of spontaneous magnon decay in a Néel skyrmion crystal sheet. Arrows in the background indicate the magnetic texture of the SkX, with color indicating the in-plane component of the texture. White spheres and wavy lines indicate propagating magnons. A magnon incoming from the left spontaneously decays into two other magnons. This number nonconserving process is brought about by the noncollinear texture and gives rise to a zero-temperature quantum damping of the SkX's eigenmodes. 


\section{THEORY}

Interacting spins $\hat{\boldsymbol{S}}_{\boldsymbol{r}}$, localized at lattice sites with position vector $\boldsymbol{r}$, are effectively described by spin Hamiltonians $\hat{H}=\frac{1}{2} \sum_{\boldsymbol{r}, \boldsymbol{r}^{\prime}} \hat{\boldsymbol{S}}_{\boldsymbol{r}} \cdot \mathbb{I}_{\boldsymbol{r}, \boldsymbol{r}^{\prime}} \cdot \hat{\boldsymbol{S}}_{\boldsymbol{r}^{\prime}}+b \sum_{\boldsymbol{r}} \hat{S}_{\boldsymbol{r}}^{z}$, with interaction matrices $\mathbb{I}_{r, r^{\prime}}$ and a Zeeman energy due to magnetic field $b$. The classical ground state of $\hat{H}$, obtained by treating the $\hat{\boldsymbol{S}}_{\boldsymbol{r}}$ 's as classical vectors $\boldsymbol{S}_{\boldsymbol{r}}$ in $\mathbb{R}^{3}$, defines local reference frames $\left\{\boldsymbol{e}_{r}^{\boldsymbol{x}}, \boldsymbol{e}_{r}^{y}, \boldsymbol{e}_{r}^{z}\right\}$, with $\boldsymbol{e}_{r}^{z}$ along the classical ground-state direction. Excitations above this ground state are captured by the Holstein-Primakoff transformation [111] to bosons $\hat{a}_{r}^{(\dagger)}: \hat{S}_{\boldsymbol{r}}=\sqrt{S}\left(\hat{f}_{\boldsymbol{r}} \hat{a}_{\boldsymbol{r}} \boldsymbol{e}_{\boldsymbol{r}}^{-}+\hat{a}_{\boldsymbol{r}}^{\dagger} \hat{f}_{\boldsymbol{r}} \boldsymbol{e}_{\boldsymbol{r}}^{+}\right)+\left(S-\hat{a}_{\boldsymbol{r}}^{\dagger} \hat{a}_{\boldsymbol{r}}\right) \boldsymbol{e}_{\boldsymbol{r}}^{z}$, with $\boldsymbol{e}_{\boldsymbol{r}}^{ \pm}=$ $\left(\boldsymbol{e}_{r}^{x} \pm i \boldsymbol{e}_{r}^{y}\right) / \sqrt{2}$ and spin length $S$. Expanding $\hat{f}_{r}=(1-$ $\left.\hat{a}_{r}^{\dagger} \hat{a}_{r} / 2 S\right)^{1 / 2}$ in powers of $1 / S$ leads to $\hat{H}=\sum_{t=0}^{\infty} \hat{H}_{t}$, where $\hat{H}_{t} \propto O\left(S^{2-t / 2}\right)$ includes bosonic operators to the $t$ th power. Sub-Hamiltonian $\hat{H}_{0}$ gives the classical ground-state energy, $\hat{H}_{1}$ vanishes if the magnetic texture is stable, $\hat{H}_{2}$ describes free magnons, and $\hat{H}_{t>2}$ denotes interactions. In particular, $\hat{H}_{3}$ comprises number nonconserving three-magnon interactions.

Often, however, linear spin-wave theory $\left(\hat{H}_{2}\right)$ is sufficient because the single-particle sector is disconnected from manyparticle sectors in one of the following limits: (1) classical spins $S \rightarrow \infty$ because interactions cause at least $1 / S$ corrections to the spectrum, (2) low temperatures $T$ that freeze out thermally activated interactions, or (3) large fields $b$ that polarize the magnet and energetically separate the $m$-magnon manifolds, whose energies grow with $m b(m=1,2, \ldots)$.

Intriguingly, none of the above limits is applicable to SkXs. (1) Flat bands cause decay singularities at twice their energy, only regularized at the same $1 / S$ order that introduces damping in the first place. Thus, there is no $1 / S$ smallness in the damping and large remnants of quantum effects in effectively classical spin systems are expected, an effect otherwise known from kagome antiferromagnets [98,99]. (2) Three-magnon interactions $\left(\hat{H}_{3}\right)$ cause spontaneous decay even at $T=0$ [95]. (3) Field polarization destroys the SkX and cannot be used.

This prima facie case for studying magnon-magnon interactions in SkXs calls for a nonlinear spin-wave theory of chiral magnets. Here we consider a two-dimensional chiral magnet on the triangular lattice (in the $x y$ plane) with lattice constant $a$. Its interaction matrices with elements $\left(\mathbb{I}_{r, r^{\prime}}\right)_{m n}=$ $-J \delta_{m n}+\epsilon_{m n p} D_{r . r^{\prime}}^{p}\left(\delta_{m n}\right.$ and $\epsilon_{m n p}$ are the Kronecker delta and the Levi-Civita symbol, respectively) include isotropic symmetric ferromagnetic exchange $J>0$ and antisymmetric exchange denoted by a Dzyaloshinskii-Moriya (DM) $[112,113]$ vector $\boldsymbol{D}_{\boldsymbol{r}, \boldsymbol{r}^{\prime}}=D \boldsymbol{e}_{z} \times \boldsymbol{e}_{\boldsymbol{r}, \boldsymbol{r}^{\prime}}$ of length $D$ that complies with interfacial inversion symmetry breaking $[114,115] ; \boldsymbol{e}_{z}$ is a unit vector in the $z$ direction and $\boldsymbol{e}_{\boldsymbol{r}, \boldsymbol{r}^{\prime}}$ one in the bond direction.

For $D \neq 0$ and $b=0$, the ground-state spin spiral has a pitch of $\lambda \approx \sqrt{3} \pi a \arctan ^{-1}(\sqrt{3} D / 2 J)$ (see Appendix A 1 ). Once $b>b_{c, 1} \approx 0.2 D^{2} / J$ [116], a Néel SkX forms. A commensurate SkX with $\lambda=M a$ ( $M$ an integer) and $N=$ $M^{2}$ spins per magnetic unit cell is obtained for $D / J \approx$ $\tan (2 \pi / M)$. Field polarization is reached at $b>b_{\mathrm{c}, 2} \approx$ $0.8 D^{2} / J[116]$.

Numerically, we proceed as follows. We choose $M$, compute $D$ and $J$, and equilibrate a random spin configuration at $T=0$. We obtain the single-particle energies $\varepsilon_{\boldsymbol{k}, v}$ by diagonalizing $\hat{H}_{2}$ in terms of normal mode bosons $\hat{b}_{\boldsymbol{k}, v}^{(\dagger)}$, where $v=1, \ldots, N$ is the band index and $\boldsymbol{k}$ the crystal momentum (see Appendix A 2). The cubic decay processes, within which a magnon in state $(\boldsymbol{p}, v)$ decays into two magnons in states $(\boldsymbol{k}, \lambda)$ and $(\boldsymbol{q}, \mu)$, respectively, read (see Appendix A 2)

$$
\hat{H}_{3}^{\mathrm{d}}=\frac{\sqrt{S}}{\sqrt{N_{\mathrm{u}}}} \sum_{\lambda, \mu, \nu} \sum_{\boldsymbol{k}, \boldsymbol{q}, \boldsymbol{p}}^{p=\boldsymbol{k} \boldsymbol{q}}\left(\frac{1}{2 ! 1 !} \mathcal{V}_{\boldsymbol{k}, \boldsymbol{q} \leftarrow \boldsymbol{p}}^{\lambda \mu \leftarrow v} \hat{b}_{\boldsymbol{k}, \lambda}^{\dagger} \hat{b}_{\boldsymbol{q}, \mu}^{\dagger} \hat{b}_{\boldsymbol{p}, \nu}+\text { H.c. }\right)
$$

( $N_{\mathrm{u}}$ the number of unit cells). The vertex $\mathcal{V}_{\boldsymbol{k}, \boldsymbol{q} \leftarrow p}^{\lambda \mu \leftarrow \nu}$ comprises the interaction strength and the Hermitian conjugate part a magnon coalescence.

To account for $\hat{H}_{3}$, we perform second-order many-body perturbation theory. Concentrating on dynamical $1 / S$ corrections to the spectrum, we approximate the single-particle Green's function by $G_{\boldsymbol{k}, v}^{-1}(\varepsilon) \approx \varepsilon-\varepsilon_{\boldsymbol{k}, v}+i \Gamma_{\boldsymbol{k}, v}$, from which we obtain the spectral function $A_{k, v}(\varepsilon) \approx-\operatorname{Im} G_{k, v}(\varepsilon) / \pi$ of band $v$. Evaluating single-bubble diagrams within the on-shell approximation, we derive the spontaneous zero-temperature damping (see Appendix A 3)

$$
\Gamma_{\boldsymbol{k}, v}^{\mathrm{spon}}=\frac{\pi}{2 N_{\mathrm{u}}} \sum_{\boldsymbol{q}} \sum_{\lambda, \mu}\left|\mathcal{V}_{\boldsymbol{q}, \boldsymbol{k}-\boldsymbol{q} \leftarrow \boldsymbol{k}}^{\lambda \mu \leftarrow \nu}\right|^{2} \delta\left(\varepsilon_{\boldsymbol{k}, \nu}-\varepsilon_{\boldsymbol{q}, \lambda}-\varepsilon_{\boldsymbol{k}-\boldsymbol{q}, \mu}\right) .
$$

\section{RESULTS}

First, we review the results of harmonic theory $[44,48,52]$. Figures 2(a)-2(c) show the dispersion of the lowest freemagnon bands $\varepsilon_{\boldsymbol{k}, \nu}$ of an $M=8 \mathrm{SkX}$ dependent on $b(D / J=$ $1)$. We identify flat modes that derive from single-skyrmion modes associated with an $l$ th-order polygon deformation in real space [117] $[l=2$, elliptic $(\mathrm{E}) ; l=3$, triangular $(\mathrm{T})$; $l=4$, quadrupolar $(\mathrm{Q}) ; l=5$, pentagonal mode $(\mathrm{P})$; etc.]. Moreover, there are dispersive modes, the lowest four of which coincide with the gyrotropic $(\mathrm{G})$, anticlockwise (A), breathing (B), and clockwise (C) modes, respectively, at the Brillouin zone center $[48,52$ ] [cf. labels in Fig. 2(d)]. The G mode derives from the translational $l=1$ mode of a single skyrmion [118]. The A, B, and C modes carry a nontrivial Chern number and support chiral edge magnons $[44,48,52]$.

The color of the bands in Figs. 2(a)-2(c) indicates the magnonic out-of-plane magnetic moment $\mu_{k, v}=-\partial \varepsilon_{k, v} / \partial b$. A positive (negative) $\mu_{k, v}$ means that an increasing field shifts the modes towards lower (higher) energies. Flat modes carry large negative $\mu_{k, v}$ because of additional angular momentum associated with $l$. Hence, upon increasing the field, they overtake the dispersive modes, which strongly influences SQD. To see so, note that $\Gamma_{\boldsymbol{k}, v}^{\text {spon }}$ is a weighted two-magnon $\operatorname{DOS} \mathcal{D}_{\boldsymbol{k}}^{2 \mathrm{~m}}(\varepsilon)=\frac{1}{N_{u}} \sum_{\boldsymbol{q}} \sum_{\lambda, \mu} \delta\left(\varepsilon-\varepsilon_{\boldsymbol{q}, \lambda}-\varepsilon_{\boldsymbol{k}-\boldsymbol{q}, \mu}\right)$ at $\varepsilon=\varepsilon_{\boldsymbol{k}, \nu}$. In Figs. 2(d)-2(f) the $\varepsilon_{\boldsymbol{k}, v}$ 's are overlaid with colormaps that show $\mathcal{D}_{k}^{2 \mathrm{~m}}(\varepsilon)$. Besides extended regions of moderate $\mathcal{D}_{k}^{2 \mathrm{~m}}(\varepsilon)$, several horizontal features, i.e., sharp yellow lines, at twice the energy (or at the sum of two energies) of flat modes are identified. If a single-magnon branch crosses a region of large $\mathcal{D}_{k}^{2 \mathrm{~m}}(\varepsilon)$, it is "in resonance" with flat modes and the kinematically allowed phase space for SQD, i.e., the number of decay channels that fulfill both energy and momentum conservation, 


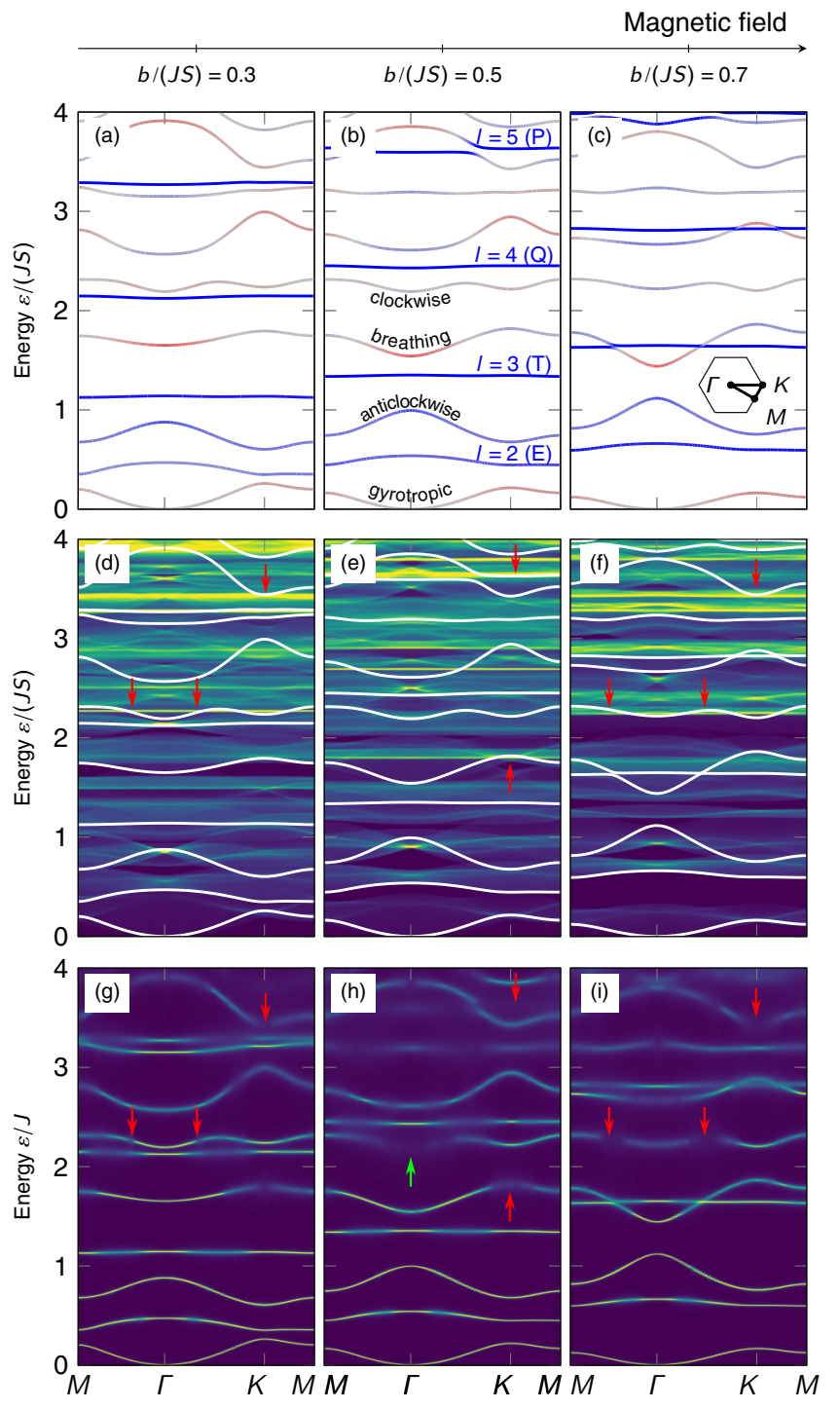

FIG. 2. Low-energy portion of the $M=8 \mathrm{SkX}$ magnon spectrum dependent on magnetic field $b$. (a)-(c) Single-particle magnon energies $\varepsilon_{\boldsymbol{k}, v}$, whose blue/gray/red color encodes negative/zero/positive magnetic moment $\mu_{k, v}$. (d)-(f) Two-magnon DOS $\mathcal{D}_{k}^{2 \mathrm{~m}}(\varepsilon)$ (blue, zero; yellow, maximal), with red arrows indicating selected points where $\varepsilon_{\boldsymbol{k}, v}$ (white lines) crosses regions of large $\mathcal{D}_{\boldsymbol{k}}^{2 \mathrm{~m}}(\varepsilon)$. (g)-(i) Spectral function $A_{k}(\varepsilon)$ for $S=1$, with sharp yellow/broad blue quasiparticle peaks indicating undamped/damped magnons. Strong damping is found for the crossing points (red arrows).

is particularly large. Selected instances of such crossings are marked by red arrows and pronounced $\Gamma_{\boldsymbol{k}, \boldsymbol{v}}^{\text {spon }}$ is expected.

We confirm this prediction by calculating $\Gamma_{\boldsymbol{k}, \nu}^{\text {spon }}$ and $A_{k}(\varepsilon)=\sum_{\nu=1}^{N} A_{k, v}(\varepsilon)$, the latter of which is shown in Figs. 2(g)-2(i). Stable magnons appear as sharp yellow quasiparticle peaks, while strongly damped magnons experience considerable lifetime broadening (broad blue features). For example, consider the regions of pronounced damping marked by red arrows in Fig. 2(g), which can be traced back to $\mathcal{D}_{k}^{2 \mathrm{~m}}(\varepsilon)$ in Fig. 2(d). These flat-band resonances can be so large that the spectral weight is almost completely wiped out, as exemplified by the pentagonal $l=5$ mode at $b / J S=0.5$
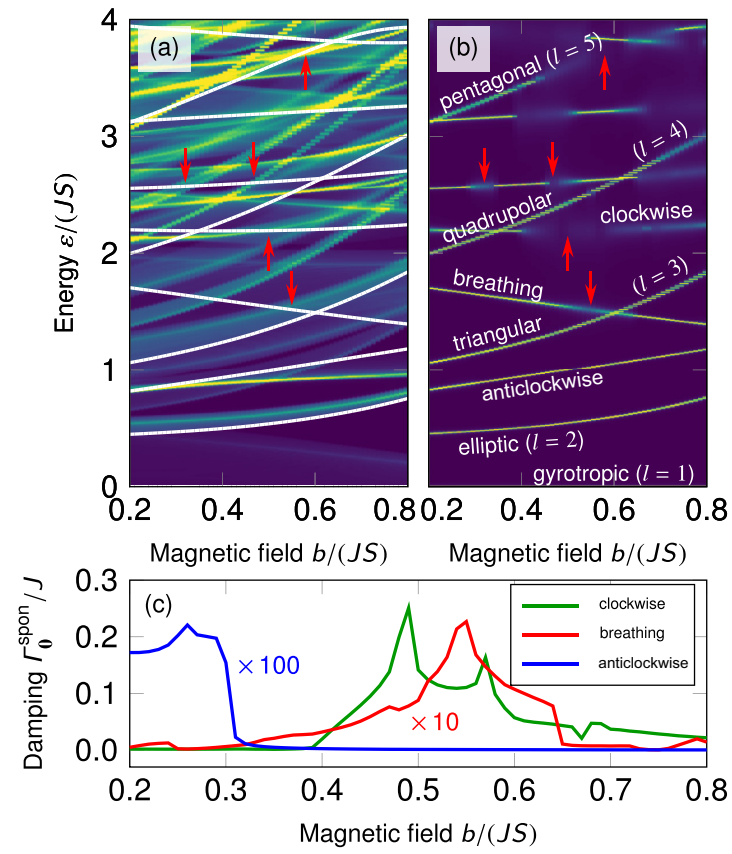

FIG. 3. Magnon decay at the Brillouin zone center $(\boldsymbol{k}=\mathbf{0})$ of an $M=8 \mathrm{SkX}$ dependent on magnetic field $b$. (a) Two-magnon DOS $\mathcal{D}_{0}^{2 \mathrm{~m}}(\varepsilon)$ (blue, zero; yellow, maximal), with red arrows indicating selected points where the eigenmode energies $\varepsilon_{\mathbf{0}, v}$ (white lines) cross regions of large $\mathcal{D}_{0}^{2 \mathrm{~m}}(\varepsilon)$. (b) Spectral function $A_{0}(\varepsilon)$ for $S=1$, with sharp yellow/broad blue quasiparticle peaks indicating undamped/damped magnons. Strong damping is found for the crossing points (red arrows). (c) Spontaneous damping $\Gamma_{\mathbf{0}, v}^{\text {spon }}$ of the three magnetically active modes reveals that the clockwise (anticlockwise) mode is the least (most) stable.

in Figs. 2(b), 2(e), and 2(h) (cf. the upper right red arrow). However, a slight magnetic-field detuning of the resonance condition leads to a reappearing quasiparticle peak. To confirm so, see Appendix B 1 .

Besides flat-mode resonances, one identifies several regions with large damping but moderate $\mathcal{D}_{k}^{2 \mathrm{~m}}(\varepsilon)$, associated with decays into (at least) one dispersive mode. As an example, consider the $\mathrm{C}$ mode in Fig. 2(h), indicated by the green arrow. Its strong damping is not related to a particularly large $\mathcal{D}_{k}^{2 \mathrm{~m}}(\varepsilon)$ in Fig. 2(e). This mechanism is the prime cause of high-energy magnon damping in Figs. 2(g)-2(i), reflecting the abundance of kinematically allowed decay channels.

Since all decay channels that contain at least one flat mode as decay product exhibit a strong field dependence, the SQD is field tunable and highly energy selective, which sets SkXs apart from other SQD platforms. This finding can be confirmed by inelastic neutron or resonant x-ray-scattering experiments, which measure the dynamical structure factor [119]. A rich magnetic-field dependence of linewidths is expected. To pursue this thought further, we continue with an analysis of uniform excitations $(\boldsymbol{k}=\mathbf{0})$, which can even be studied by absorption experiments [120-123]. For the magnetic field normal to the SkX, only the ABC modes are active [23]. However, inclined fields admit additional active modes [124]. 


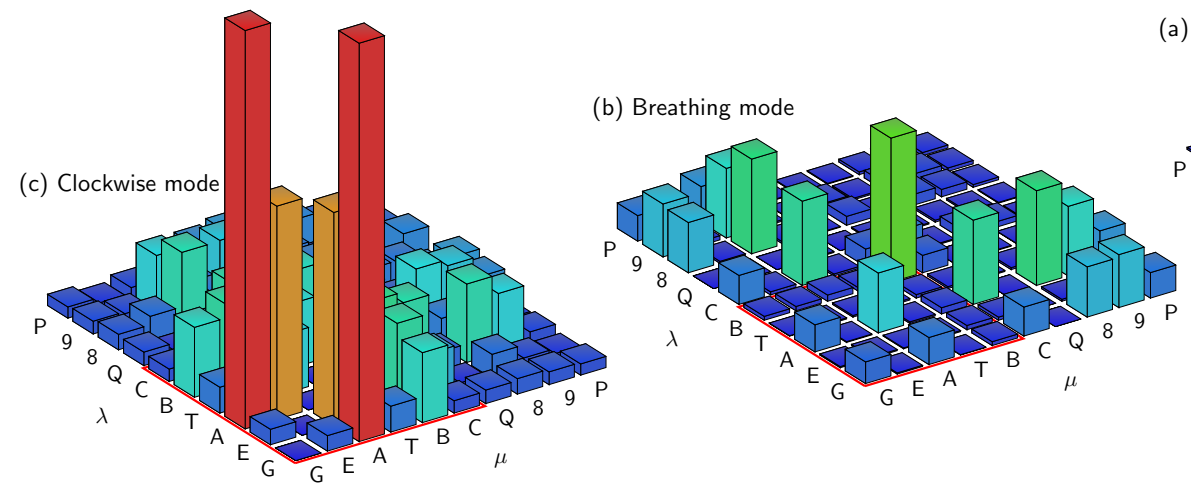

(a) Anticlockwise mode
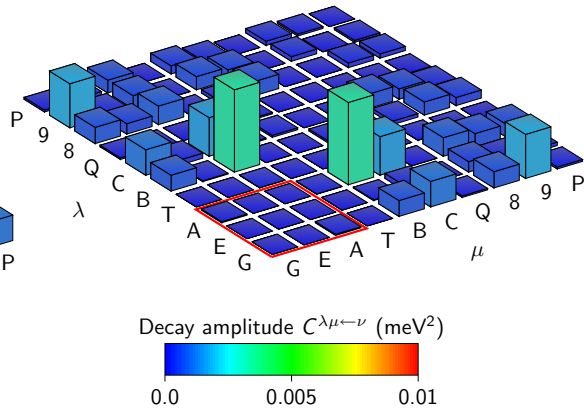

FIG. 4. Decay channel analysis of an $M=8 \mathrm{SkX}$ at $b / J S=0.5$ and $\boldsymbol{k}=\mathbf{0}$. Histograms show the decay amplitude $C^{\lambda \mu \leftarrow v}$ for $\lambda$, $\mu=$ $1, \ldots, 10$ and (a) $v=3$, anticlockwise mode (A); (b) $v=5$, breathing mode (B); and (c) $v=6$, clockwise mode (C). The histograms are symmetric due to decay product symmetry $(\lambda \leftrightarrow \mu$; cf. Appendix A 2$)$. Red squares indicate the subsection of decay channels that could, in principle, fulfill energy conservation. By comparing the amplitudes of these channels between the three modes, the clockwise (anticlockwise) mode is found to interact strongly (weakly) with other modes. This explains the two order of magnitude difference in the damping [cf. Fig. 3(c)].

Figure 3(a) shows $\mathcal{D}_{\mathbf{0}}^{2 \mathrm{~m}}(\varepsilon)$ dependent on $b$. Again, strong damping is found when $\varepsilon_{\mathbf{0}}, v$ crosses a region of large $\mathcal{D}_{\mathbf{0}}^{2 \mathrm{~m}}(\varepsilon)$. Several such encounters are highlighted by red arrows, from which a rich structure of strong quasiparticle peak broadening is expected. Indeed, $A_{0}(\varepsilon)$ in Fig. 3(b) reveals that high-energy magnons exhibit field intervals within which their quasiparticle peak disappears and reappears. In contrast, low-energy magnons exhibit negligible quantum damping.

There are considerable differences in damping between the ABC modes [cf. Fig. 3(c)]. The SQD is particularly strong for the $\mathrm{C}$ mode, whose damping $(\lesssim 0.2 J)$ is on par with frustrated quantum antiferromagnets $[84,88,95]$. In contrast, the damping of the B (A) mode is a factor of 10 (100) smaller. These differences cannot solely be explained in terms of $\mathcal{D}_{\mathbf{0}}^{2 \mathrm{~m}}(\varepsilon)$ but must be looked for in $\mathcal{V}_{\boldsymbol{k}, \boldsymbol{q} \leftarrow \boldsymbol{p}}^{\lambda \mu}$.

We analyze each decay channel $v \rightarrow(\lambda, \mu)$ by measuring its integrated decay amplitude $C^{\lambda \mu \leftarrow v} \equiv \frac{1}{N_{\mathrm{u}}} \sum_{\boldsymbol{q}}\left|\mathcal{V}_{\boldsymbol{q},-\boldsymbol{q} \leftarrow \mathbf{0}}^{\lambda \mu \leftarrow v}\right|^{2}$. It encodes dynamical rather than kinematic details. Results for 1000 decay channels, involving the ten lowest modes at $b / J S=0.5$, are presented in Appendix B 2. Here we focus on the decay of ABC modes (Fig. 4).

The A mode $[v=3$, Fig. 4(a) $]$ exhibits a small interaction with other modes, especially those that could possibly fulfill energy conservation ( $\mathrm{G}$ and $\mathrm{E}$ ). Thus, the $\mathrm{A}$ mode is the most stable out of the ABC modes for both kinematic and dynamic reasons. The B mode $[v=5$, Fig. $4(\mathrm{~b})]$ prefers channel $\mathrm{B} \rightarrow(\mathrm{B}, \mathrm{B})$, which, however, can never obey energy conservation, and channels involving the $\mathrm{G}$ and/or $\mathrm{A}$ mode, causing the moderate damping found in Fig. 3. In contrast, channels with polygon modes $\mathrm{E}, \mathrm{T}$, and $\mathrm{Q}$ as decay products are suppressed by two orders of magnitude. Finally, being highest in energy, the $\mathrm{C}$ mode $[v=6$, Fig. $4(\mathrm{c})]$ has several kinematic possibilities to decay. On top of that are pronounced instabilities towards the $\mathrm{A}$ and $\mathrm{B}$ modes. Thus, the $\mathrm{C}$ mode is the least stable among the ABC modes. These findings are corroborated by the nontrivial $b$ dependence of the $\mathrm{ABC}$ modes' interaction strengths (see Appendix B 3): While that of the $\mathrm{C}$ mode (and B mode) increases as the field increases, that of the A mode decreases.
The results discussed so far apply to zero temperature. In Appendix B 4 we extend our analysis to finite temperatures: At $k_{\mathrm{B}} T / J=0.2$ and $b / J S=0.485$ ( $k_{\mathrm{B}}$ the Boltzmann constant) the thermal damping of the $\mathrm{C}$ mode is still only half of its quantum damping. Thus, even the quantum dynamics of SkXs stabilized by moderate thermal fluctuations may be dominated by SQD. However, for consistency of spin-wave theory, $k_{\mathrm{B}} T \ll J S$ must hold (see Appendix B 5).

\section{DISCUSSION AND CONCLUSION}

Spontaneously decaying magnons cause field-tunable intrinsic zero-temperature damping of SkX eigenmodes. For applications in topological magnonics, this damping must not be larger than the average band gap $\Delta \approx 12 S J / N \approx$ $0.3\left[J S(D / J)^{2}\right]$, hosting chiral edge states. With the topologically nontrivial ABC modes' damping $\Gamma_{X}^{\text {spon }} \approx F_{X} J(D / J)^{4}$, with $X \in\{\mathrm{A}, \mathrm{B}, \mathrm{C}\}$ and $F_{\mathrm{A}}=10^{-3}, F_{\mathrm{B}}=10^{-2}$, and $F_{\mathrm{C}}=$ $10^{-1}$ (see Appendix B 6), the crucial ratio reads $\Gamma_{X}^{\text {spon }} / \Delta \approx$ $F_{X} /(0.3 S)(D / J)^{2} \ll 1$ for typical values $D / J \ll 1$. For thin films of the skyrmion-hosting compound $\mathrm{Cu}_{2} \mathrm{OSeO}_{3}$, we estimate $\Gamma_{\mathrm{C}}^{\text {spon }} / \Delta \approx 5.6 \mathrm{neV} / 4.7 \mu \mathrm{eV}=1.2 \times 10^{-3}$ using $S=1, J / k_{\mathrm{B}} \approx 50 \mathrm{~K}$, and $D / J \approx 0.06$ [8]. Even for small skyrmions $(D / J=1)$, quantum damping of the $\mathrm{A}$ and $\mathrm{B}$ modes is negligible. Hence, SkXs are an excellent platform for low-energy topological magnonics, which is at variance with other topological magnets [125-127] whose chiral edge magnons appear at high energies of the order of the exchange energy.

At higher energies, SkXs exhibit considerable SQD, which yields a quantum correction to the classical damping $\Gamma_{k, v}^{\alpha} \approx$ $\alpha \varepsilon_{k, v} \propto O(S)$ due to phenomenological Gilbert damping $\alpha$. Skyrmion crystals are unique in that not only neutron scattering but also magnetic resonance experiments can address the lifetime broadening due to SQD. For the magnetically active $\mathrm{C}$ mode, with energy $\varepsilon_{\mathrm{C}} \sim J S(D / J)^{2}$, the relative importance of quantum to classical damping reads $R_{\mathrm{C}} \equiv \Gamma_{\mathrm{C}}^{\text {spon }} / \Gamma_{\mathrm{C}}^{\alpha} \approx$ $10^{-1}(\alpha S)^{-1}(D / J)^{2}$. Since ultralow $\alpha \sim 10^{-4}-10^{-3}$ is possible in both metals [128-137] and insulators [138], quantum damping may dominate over classical damping $\left(R_{\mathrm{C}}>1\right)$, e.g., 
$R_{\mathrm{C}} \approx 3.6$ in $\mathrm{Cu}_{2} \mathrm{OSeO}_{3}$ with $\alpha=10^{-4}$ [138], a prediction that may be experimentally tested in the recently discovered low-temperature SkX [139].

Our strictly two-dimensional considerations apply both to thin films (cf. Appendix B 7) and quasi-two-dimensional layered structures, e.g., van der Waals magnets [140-142] and Janus layers $[143,144]$. Although we concentrated on Néel skyrmions stabilized by interfacial DM interaction, our results can be carried over one to one to Bloch skyrmions due to bulk DM interaction because neither the harmonic magnon bands nor their damping is influenced by the skyrmion helicity. Additional anisotropies, exchange frustration [49,145-151], and dipolar and four-spin interactions [152] are expected to merely quantitatively renormalize the quantum damping.

\section{ACKNOWLEDGMENTS}

We thank Sebastián Díaz for helpful discussions. This work was supported by the Georg H. Endress Foundation, the Swiss National Science Foundation, and NCCR QSIT. This project received funding from the European Union's Horizon 2020 research and innovation program (ERC Starting Grant Agreement No. 757725).

\section{APPENDIX A: DETAILS OF THEORETICAL DERIVATIONS}

\section{Classical magnetic ground state}

Before studying the dynamics of a spin Hamiltonian $\hat{H}$, we need to find the classical magnetic ground state, assuming that spin operators $\hat{\boldsymbol{S}}_{\boldsymbol{r}}$ are replaced by spin vectors $\boldsymbol{S}_{\boldsymbol{r}}$ in $\mathbb{R}^{3}$. For $J>0$, neighboring spins prefer ferromagnetic alignment that competes with the spiralization tendency due to DM interaction. In the absence of a magnetic field, the classical magnetic ground state is thus a spin spiral. To determine its pitch we perform a spatial Fourier transformation from coordinates $\boldsymbol{r}$ to (crystal) momenta $\boldsymbol{k}$,

$$
S_{r}=\frac{1}{\sqrt{N_{\mathrm{s}}}} \sum_{k} e^{i k \cdot r} S_{k},
$$

where $N_{\mathrm{s}}$ is the total number of spins. Thus, the classical ground-state energy in zero field is recast as

$$
\begin{aligned}
H & =\frac{1}{2} \sum_{\boldsymbol{r}, \boldsymbol{r}^{\prime}} \boldsymbol{S}_{\boldsymbol{r}} \cdot \mathbb{I}_{\boldsymbol{r}, \boldsymbol{r}^{\prime}} \cdot \boldsymbol{S}_{\boldsymbol{r}^{\prime}} \\
& =\frac{1}{2 N_{\mathrm{s}}} \sum_{\boldsymbol{k}, \boldsymbol{k}^{\prime}} \sum_{\boldsymbol{r}, \Delta \boldsymbol{r}} \boldsymbol{S}_{\boldsymbol{k}} \cdot\left(e^{i\left(\boldsymbol{k}+\boldsymbol{k}^{\prime}\right) \cdot \boldsymbol{r}} e^{i \boldsymbol{k}^{\prime} \cdot \boldsymbol{\Delta r}} \mathbb{I}_{\boldsymbol{r}, \boldsymbol{r}+\boldsymbol{\Delta r} \boldsymbol{r}}\right) \cdot \boldsymbol{S}_{\boldsymbol{k}^{\prime}} \\
& =\frac{1}{2} \sum_{\boldsymbol{k}} \boldsymbol{S}_{\boldsymbol{k}} \cdot \underbrace{\left(\sum_{\Delta \boldsymbol{r}} e^{-i \boldsymbol{k} \cdot \boldsymbol{\Delta} \boldsymbol{r}} \mathbb{I}_{\Delta \boldsymbol{r}}\right)}_{\mathbb{I}_{\boldsymbol{k}}} \cdot \boldsymbol{S}_{-\boldsymbol{k}} .
\end{aligned}
$$

We use $\sum_{r} e^{i\left(k+k^{\prime}\right) \cdot r}=N_{\mathrm{s}} \delta_{\boldsymbol{k},-\boldsymbol{k}^{\prime}}$ and $\boldsymbol{r}^{\prime}=\boldsymbol{r}+\boldsymbol{\Delta r}$, introducing the distance vector $\boldsymbol{\Delta} \boldsymbol{r}=\boldsymbol{r}^{\prime}-\boldsymbol{r}$. We set $\mathbb{I}_{\boldsymbol{r}, \boldsymbol{r}^{\prime}}=\mathbb{I}_{\boldsymbol{r}, \boldsymbol{r}+\boldsymbol{\Delta} \boldsymbol{r}}=$ $\mathbb{I}_{\boldsymbol{\Delta} r}$, reflecting spatial homogeneity of magnetic interactions.

Since magnetic interactions take place only between nearest neighbors, the Fourier kernel of the classical ground-state

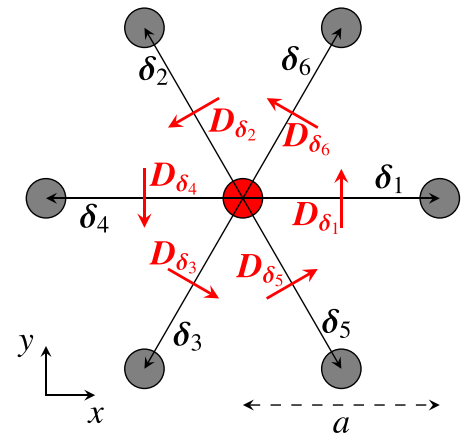

FIG. 5. Triangular lattice with indicated nearest-neighbor bond vectors $\boldsymbol{\delta}_{i}$ and respective DM vectors $\boldsymbol{D}_{\delta_{i}}(i=1, \ldots, 6)$. The lattice constant is $a$.

energy can be rewritten as

$$
\mathbb{I}_{\boldsymbol{k}}=\sum_{i=1}^{6} e^{-i \boldsymbol{k} \cdot \boldsymbol{\delta}_{i}} \mathbb{I}_{\delta_{i}},
$$

where the $\delta_{i}$ 's are the vectors to the six nearest neighbors on the triangular lattice, as shown in Fig. 5; explicitly they read

$$
\boldsymbol{\delta}_{1}=a\left(\begin{array}{l}
1 \\
0
\end{array}\right), \quad \boldsymbol{\delta}_{2}=a\left(\begin{array}{c}
-\frac{1}{2} \\
\frac{\sqrt{3}}{2}
\end{array}\right), \quad \boldsymbol{\delta}_{3}=a\left(\begin{array}{c}
-\frac{1}{2} \\
-\frac{\sqrt{3}}{2}
\end{array}\right),
$$

$\boldsymbol{\delta}_{4}=-\boldsymbol{\delta}_{1}, \boldsymbol{\delta}_{5}=-\boldsymbol{\delta}_{2}$, and $\boldsymbol{\delta}_{6}=-\boldsymbol{\delta}_{3}$. (We denoted the lattice constant by $a$.) The respective interaction matrix

$$
\mathbb{I}_{\delta_{i}}=\left(\begin{array}{ccc}
-J & 0 & -D_{\delta_{i}}^{y} \\
0 & -J & D_{\delta_{i}}^{x} \\
D_{\delta_{i}}^{y} & -D_{\delta_{i}}^{x} & -J
\end{array}\right)
$$

contains the DM vectors

$$
\boldsymbol{D}_{\delta_{1}}=D\left(\begin{array}{l}
0 \\
1
\end{array}\right), \quad \boldsymbol{D}_{\delta_{2}}=D\left(\begin{array}{c}
-\frac{\sqrt{3}}{2} \\
-\frac{1}{2}
\end{array}\right), \quad \boldsymbol{D}_{\delta_{3}}=D\left(\begin{array}{c}
\frac{\sqrt{3}}{2} \\
-\frac{1}{2}
\end{array}\right),
$$

$\boldsymbol{D}_{\delta_{4}}=-\boldsymbol{D}_{\delta_{1}}, \boldsymbol{D}_{\delta_{5}}=-\boldsymbol{D}_{\delta_{2}}$, and $\boldsymbol{D}_{\delta_{6}}=-\boldsymbol{D}_{\delta_{3}}$. They are indicated by red arrows in Fig. 5 .

To find the energy-minimizing spin configuration, we determine the eigenvalues of

$$
\mathbb{I}_{k}=\left(\begin{array}{ccc}
-Z_{k} & 0 & -Y_{k} \\
0 & -Z_{k} & X_{k} \\
Y_{k} & -X_{k} & -Z_{k}
\end{array}\right),
$$

which read

$$
V_{0, k}=-Z_{k}, \quad V_{ \pm, k}=-Z_{k} \pm \sqrt{\left|X_{k}\right|^{2}+\left|Y_{k}\right|^{2}},
$$

with

$$
\begin{aligned}
& Z_{\boldsymbol{k}}=2 J \sum_{i=1}^{3} \cos \left(\boldsymbol{k} \cdot \boldsymbol{\delta}_{i}\right), \\
& X_{\boldsymbol{k}}=-2 i \sum_{i=1}^{3} D_{\boldsymbol{\delta}_{i}}^{x} \sin \left(\boldsymbol{k} \cdot \boldsymbol{\delta}_{i}\right), \\
& Y_{\boldsymbol{k}}=-2 i \sum_{i=1}^{3} D_{\boldsymbol{\delta}_{i}}^{y} \sin \left(\boldsymbol{k} \cdot \boldsymbol{\delta}_{i}\right) .
\end{aligned}
$$



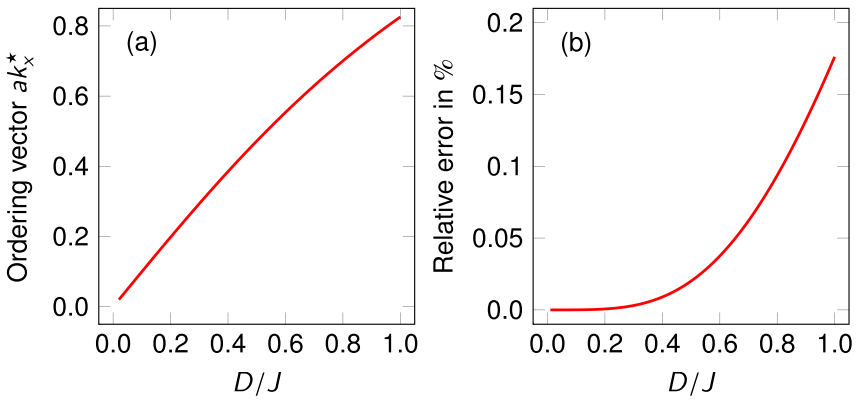

FIG. 6. (a) Spin spiral ordering vector $a k_{x}^{\star}$ dependent on $D / J$ (a lattice constant). (b) Relative error between the exact solution for $k_{x}^{\star}$ and the approximation in Eq. (A10) dependent on $D / J$.

The ordering vector $\boldsymbol{k}^{\star}$ of the ground-state texture is given by the minima of the lowest eigenvalue $V_{-, k}$. Due to the sixfold rotational symmetry of the triangular lattice, there are six minima; one solution reads $\boldsymbol{k}^{\star}=\left(k_{x}^{\star}, 0\right)$, with the others to be obtained by rotation. The numerically obtained solution for $k_{x}^{\star}$, shown in Fig. 6(a) dependent on $D / J$, is very well approximated by

$$
k_{x}^{\star} \approx \frac{2}{\sqrt{3} a} \arctan \left(\frac{\sqrt{3}}{2} \frac{D}{J}\right) .
$$

Figure 6(b) shows the relative error between Eq. (A10) and the exact solution. Even for ratios as large as $D / J=1$, the relative error is smaller than $0.2 \%$. From Eq. (A10), we obtain a spin spiral pitch of

$$
\lambda=\frac{2 \pi}{k_{x}^{\star}} \approx \sqrt{3} \pi a\left[\arctan \left(\frac{\sqrt{3}}{2} \frac{D}{J}\right)\right]^{-1} .
$$

Once the magnetic field is larger than a critical value, the skyrmion crystal is approximately formed as a superposition of a ferromagnetic component and three fundamental spin spirals (neglecting higher harmonics), each pair of which encloses an angle of $\pm 2 \pi / 3$. Restricting our focus on commensurate textures, we require $\lambda=M a$ to be an integer multiple of $a$; thus $M \in \mathbb{N}$. It follows that if a skyrmion crystal with $N=M^{2}$ spins per magnetic unit cell (i.e., per skyrmion) is desired, the magnetic interaction parameters must obey

$$
\frac{D}{J} \approx \frac{2}{\sqrt{3}} \tan \left(\frac{\sqrt{3} \pi}{M}\right) \approx \tan \left(\frac{2 \pi}{M}\right) .
$$

We note in passing that we use a triangular lattice because it naturally lends itself to a description of a hexagonal skyrmion crystal. Nonetheless, similar results are expected for other two-dimensional lattices, e.g., the square lattice. The details of the structural lattice are irrelevant as long as a single skyrmion's diameter is large compared to the structural lattice constant.

Now that we approximately know the size of the skyrmion unit cell $(M \times M$ spins $)$ for a given $D / J$ ratio, the actual skyrmion texture within the unit cell is determined numerically in a finite magnetic field. Starting from a random spin configuration, we alternate between microcanonical overrelaxation steps and Landau-Lifshitz-Gilbert (LLG) steps. The former update each spin according to

$$
\boldsymbol{S}_{r}^{\text {new }}=2 \frac{\boldsymbol{B}_{r} \cdot \boldsymbol{S}_{r}^{\text {old }}}{\left|\boldsymbol{B}_{r}\right|^{2}} \boldsymbol{B}_{\boldsymbol{r}}-\boldsymbol{S}_{\boldsymbol{r}}^{\text {old }}
$$

with $\boldsymbol{B}_{\boldsymbol{r}}=-\partial H / \partial \boldsymbol{S}_{\boldsymbol{r}}$ the effective magnetic field at site $\boldsymbol{r}$. For the latter, we integrate the LLG equation without a precession term, i.e.,

$$
\dot{\boldsymbol{S}}_{\boldsymbol{r}}=-\frac{\alpha}{1+\alpha^{2}} \boldsymbol{S}_{\boldsymbol{r}} \times\left(\boldsymbol{S}_{\boldsymbol{r}} \times \boldsymbol{B}_{r}\right),
$$

utilizing the Heun integration scheme [153]. We work in the overdamped limit with a Gilbert damping $\alpha=0.99$.

\section{Spin-to-boson transformation}

Once the magnetic ground state with $N$ spins per unit cell is found, we assign a coordinate system $\left\{\boldsymbol{e}_{\mu}^{x}, \boldsymbol{e}_{\mu}^{y}, \boldsymbol{e}_{\mu}^{z}\right\}$ to each basis site $(\mu=1, \ldots, N)$. The local reference frames are chosen such that $\boldsymbol{e}_{\mu}^{z}$ points along the local ground-state direction of spin $S_{\boldsymbol{r}+\boldsymbol{r}_{\mu}}$. Here $\boldsymbol{r}$ is the position vector to the magnetic unit cell and $\boldsymbol{r}_{\mu}$ is the position vector to the $\mu$ th basis site within the unit cell. Then the spin Hamiltonian may be recast as

$$
\hat{H}=\frac{1}{2} \sum_{\boldsymbol{r}, \boldsymbol{r}^{\prime}} \sum_{\boldsymbol{r}_{\mu}, \boldsymbol{r}_{v}} \hat{\boldsymbol{S}}_{\boldsymbol{r}+\boldsymbol{r}_{\mu}} \cdot \mathbb{I}_{\boldsymbol{r}+\boldsymbol{r}_{\mu}, \boldsymbol{r}^{\prime}+\boldsymbol{r}_{v}} \cdot \hat{\boldsymbol{S}}_{\boldsymbol{r}^{\prime}+\boldsymbol{r}_{v}}+\sum_{\boldsymbol{r}} \sum_{\boldsymbol{r}_{\mu}} \boldsymbol{b} \cdot \hat{\boldsymbol{S}}_{\boldsymbol{r}+\boldsymbol{r}_{\mu}} .
$$

For the sake of generality, we allow for a magnetic field $\boldsymbol{b}$ in an arbitrary direction. Here $\mathbb{I}_{\boldsymbol{r}+\boldsymbol{r}_{\mu}, \boldsymbol{r}^{\prime}+\boldsymbol{r}_{\nu}}$ is the interaction matrix between the $\mu$ th and $v$ th spins belonging to the magnetic unit cells at $\boldsymbol{r}$ and $\boldsymbol{r}^{\prime}$, respectively.

We proceed with a Holstein-Primakoff (HP) transformation

$$
\begin{aligned}
\hat{\boldsymbol{S}}_{\boldsymbol{r}+\boldsymbol{r}_{\mu}}= & \sqrt{S}\left(\hat{f}_{\boldsymbol{r}+\boldsymbol{r}_{\mu}} \hat{a}_{\boldsymbol{r}+\boldsymbol{r}_{\mu}} \boldsymbol{e}_{\mu}^{-}+\hat{a}_{\boldsymbol{r}+\boldsymbol{r}_{\mu}}^{\dagger} \hat{f}_{\boldsymbol{r}+\boldsymbol{r}_{\mu}} \boldsymbol{e}_{\mu}^{+}\right) \\
& +\left(S-\hat{a}_{\boldsymbol{r}+\boldsymbol{r}_{\mu}}^{\dagger} \hat{a}_{\boldsymbol{r}+\boldsymbol{r}_{\mu}}\right) \boldsymbol{e}_{\mu}^{z},
\end{aligned}
$$

where $\boldsymbol{e}_{\mu}^{ \pm}=\left(\boldsymbol{e}_{\mu}^{x} \pm i \boldsymbol{e}_{\mu}^{y}\right) / \sqrt{2}$ and $S$ is the spin length. The bosonic operators obey the usual commutation relation $\left[\hat{a}_{\boldsymbol{r}+\boldsymbol{r}_{\mu}}, \hat{a}_{\boldsymbol{r}^{\prime}+\boldsymbol{r}_{v}}^{\dagger}\right]=\delta_{\boldsymbol{r}, r^{\prime}} \delta_{\boldsymbol{r}_{\mu}, \boldsymbol{r}_{v}}$. A Taylor expansion of

$$
\hat{f}_{\boldsymbol{r}+\boldsymbol{r}_{\mu}}=\left(1-\frac{\hat{a}_{\boldsymbol{r}+\boldsymbol{r}_{\mu}}^{\dagger} \hat{a}_{\boldsymbol{r}+\boldsymbol{r}_{\mu}}}{2 S}\right)^{1 / 2}=1-\frac{1}{2} \frac{\hat{a}_{\boldsymbol{r}+\boldsymbol{r}_{\mu}}^{\dagger} \hat{a}_{\boldsymbol{r}+\boldsymbol{r}_{\mu}}}{2 S}-\cdots
$$

leads to $\hat{H}=\hat{H}_{0}+\hat{H}_{1}+\hat{H}_{2}+\hat{H}_{3}+\hat{H}_{4}+\cdots$, with subscripts denoting the number of bosonic operators. Mathematically, the HP transformation is an expansion in the order of $1 / \sqrt{S}$, which can be made explicit by writing

$$
\hat{H}=S\left(S \hat{H}_{0}^{\prime}+\sqrt{S} \hat{H}_{1}^{\prime}+\hat{H}_{2}^{\prime}+\frac{1}{\sqrt{S}} \hat{H}_{3}^{\prime}+\frac{1}{S} \hat{H}_{4}^{\prime}+\cdots\right) .
$$

The primed $\hat{H}$ 's are independent of $S$. Thus, when we later speak of " $1 / S$ corrections to the spectrum," we mean corrections that are $1 / S$ relative to $\hat{H}_{2}$. Those can come both from $\hat{H}_{4}$ (within first-order perturbation theory) and $\hat{H}_{3}$ (within second-order perturbation theory). 


\section{a. Harmonic theory}

After plugging Eqs. (A17) and (A16) into Eq. (A15), the harmonic piece of $\hat{H}$ is found to read

$$
\begin{aligned}
\hat{H}_{2}= & \frac{S}{2} \sum_{\boldsymbol{r}, \boldsymbol{r}^{\prime}} \sum_{\boldsymbol{r}_{\mu}, \boldsymbol{r}_{v}}\left(\hat{a}_{\boldsymbol{r}+\boldsymbol{r}_{\mu}} \hat{a}_{\boldsymbol{r}^{\prime}+\boldsymbol{r}_{v}} \mathcal{I}_{\boldsymbol{r}+\boldsymbol{r}_{\mu}, \boldsymbol{r}^{\prime}+\boldsymbol{r}_{v}}^{--}+\hat{a}_{\boldsymbol{r}+\boldsymbol{r}_{\mu}}^{\dagger} \hat{a}_{\boldsymbol{r}^{\prime}+\boldsymbol{r}_{v}} \mathcal{I}_{\boldsymbol{r}+\boldsymbol{r}_{\mu}, \boldsymbol{r}^{\prime}+\boldsymbol{r}_{v}}^{+-}\right. \\
& +\hat{a}_{\boldsymbol{r}+\boldsymbol{r}_{\mu}} \hat{a}_{\boldsymbol{r}^{\prime}+\boldsymbol{r}_{v}}^{\dagger} \mathcal{I}_{\boldsymbol{r}+\boldsymbol{r}_{\mu}, \boldsymbol{r}^{\prime}+\boldsymbol{r}_{v}}^{-+}+\hat{a}_{\boldsymbol{r}+\boldsymbol{r}_{\mu}}^{\dagger} \hat{a}_{\boldsymbol{r}^{\prime}+\boldsymbol{r}_{v}}^{\dagger} \mathcal{I}_{\boldsymbol{r}+\boldsymbol{r}_{\mu}, \boldsymbol{r}^{\prime}+\boldsymbol{r}_{v}}^{++} \\
& \left.-\hat{a}_{\boldsymbol{r}+\boldsymbol{r}_{\mu}}^{\dagger} \hat{a}_{\boldsymbol{r}+\boldsymbol{r}_{\mu}} \mathcal{I}_{\boldsymbol{r}+\boldsymbol{r}_{\mu}, \boldsymbol{r}^{\prime}+\boldsymbol{r}_{v}}^{z z}-\hat{a}_{\boldsymbol{r}^{\prime}+\boldsymbol{r}_{v}}^{\dagger} \hat{a}_{\boldsymbol{r}^{\prime}+\boldsymbol{r}_{v}} \mathcal{I}_{\boldsymbol{r}+\boldsymbol{r}_{\mu}, \boldsymbol{r}^{\prime}+\boldsymbol{r}_{v}}^{z z}\right) \\
& -\sum_{\boldsymbol{r}_{\mu}} \hat{a}_{\boldsymbol{r}+\boldsymbol{r}_{\mu}}^{\dagger} \hat{a}_{\boldsymbol{r}+\boldsymbol{r}_{\mu}} \boldsymbol{b} \cdot \boldsymbol{e}_{\mu}^{z},
\end{aligned}
$$

where $\mathcal{I}_{\boldsymbol{r}+\boldsymbol{r}_{\mu}, \boldsymbol{r}^{\prime}+\boldsymbol{r}_{v}}^{\xi \zeta} \equiv \boldsymbol{e}_{\mu}^{\xi} \cdot \mathbb{I}_{\boldsymbol{r}+\boldsymbol{r}_{\mu}, \boldsymbol{r}^{\prime}+\boldsymbol{r}_{v}} \cdot \boldsymbol{e}_{v}^{\zeta}$, with $\xi, \zeta \in\{+,-, z\}$. We then perform a Fourier transformation

$$
\begin{aligned}
& \hat{a}_{\boldsymbol{r}+\boldsymbol{r}_{\mu}}=\frac{1}{\sqrt{N_{\mathrm{u}}}} \sum_{\boldsymbol{k}}^{N_{\mathrm{u}}} e^{i \boldsymbol{k} \cdot\left(\boldsymbol{r}+\boldsymbol{r}_{\mu}\right)} \hat{a}_{\boldsymbol{k}, \mu}, \\
& \hat{a}_{\boldsymbol{r}+\boldsymbol{r}_{\mu}}^{\dagger}=\frac{1}{\sqrt{N_{\mathrm{u}}}} \sum_{\boldsymbol{k}}^{N_{\mathrm{u}}} e^{-i \boldsymbol{k} \cdot\left(\boldsymbol{r}+\boldsymbol{r}_{\mu}\right)} \hat{a}_{\boldsymbol{k}, \mu}^{\dagger},
\end{aligned}
$$

with $N_{\mathrm{u}}$ the number of magnetic unit cells. In compact notation, the harmonic Hamiltonian becomes

$$
\hat{H}_{2}=\frac{1}{2} \sum_{k} \hat{A}_{k}^{\dagger} \cdot \mathbb{H}_{k} \cdot \hat{A}_{k},
$$

with

$$
\hat{\boldsymbol{A}}_{\boldsymbol{k}}=\left(\hat{a}_{\boldsymbol{k}, 1}, \ldots, \hat{a}_{\boldsymbol{k}, N}, \hat{a}_{-k, 1}^{\dagger}, \ldots, \hat{a}_{-k, N}^{\dagger}\right)^{\mathrm{T}}
$$

a Nambu space vector constructed from the Fouriertransformed HP bosons associated with the $N$ basis sites in the magnetic unit cell. The $2 N \times 2 N$ linear spin-wave kernel

$$
\mathbb{H}_{k}=S\left(\begin{array}{cc}
\mathbb{M}_{k} & \mathbb{N}_{k} \\
\mathbb{N}_{k}^{\dagger} & \mathbb{M}_{-k}^{\mathrm{T}}
\end{array}\right)
$$

has entries

$$
\begin{aligned}
&\left(\mathbb{M}_{\boldsymbol{k}}\right)_{\mu \nu}=\mathcal{I}_{\boldsymbol{k}, \mu \nu}^{+-}-\delta_{\mu, \nu}\left(\frac{1}{S} \boldsymbol{b} \cdot \boldsymbol{e}_{\mu}^{z}+\sum_{\lambda} \mathcal{I}_{\mathbf{0}, \mu \lambda}^{z z}\right), \\
&\left(\mathbb{N}_{\boldsymbol{k}}\right)_{\mu \nu}=\mathcal{I}_{\boldsymbol{k}, \mu \nu}^{++} .
\end{aligned}
$$

We abbreviated

$$
\mathcal{I}_{\boldsymbol{k}, \mu \nu}^{\xi \zeta} \equiv \sum_{\boldsymbol{\Delta} \boldsymbol{r}} \mathcal{I}_{\boldsymbol{r}_{\mu}, \boldsymbol{\Delta} \boldsymbol{r}+\boldsymbol{r}_{\nu}} e^{i \boldsymbol{k} \cdot\left(\boldsymbol{\Delta}+\boldsymbol{r}_{\nu}-\boldsymbol{r}_{\mu}\right)}
$$

where the sum runs over all difference vectors $\Delta \boldsymbol{r}$ between magnetic unit cells.

The free magnon energies $\varepsilon_{\boldsymbol{k}, v}$ are obtained from a paraunitary Bogoliubov diagonalization from HP bosons to normal modes

$$
\hat{\boldsymbol{B}}_{\boldsymbol{k}}=\mathbb{P}_{\boldsymbol{k}} \hat{\boldsymbol{A}}_{\boldsymbol{k}}=\left(\hat{b}_{\boldsymbol{k}, 1}, \ldots, \hat{b}_{\boldsymbol{k}, N}, \hat{b}_{-\boldsymbol{k}, 1}^{\dagger}, \ldots, \hat{b}_{-\boldsymbol{k}, N}^{\dagger}\right)^{\mathrm{T}} .
$$

Thus, we can rewrite Eq. (A21) in the Bogoliubov eigenbasis as

$$
\hat{H}_{2}=\frac{1}{2} \sum_{\boldsymbol{k}} \underbrace{\hat{\boldsymbol{A}}_{\boldsymbol{k}}^{\dagger} \cdot \mathbb{P}_{\boldsymbol{k}}^{\dagger}}_{\hat{\boldsymbol{B}}_{\boldsymbol{k}}^{\dagger}} \underbrace{\left(\mathbb{P}_{\boldsymbol{k}}^{\dagger}\right)^{-1} \mathbb{H}_{k} \mathbb{P}_{\boldsymbol{k}}^{-1}}_{\mathbb{E}_{k}} \underbrace{\mathbb{P}_{\boldsymbol{k}} \cdot \hat{\boldsymbol{A}}_{\boldsymbol{k}}}_{\hat{\boldsymbol{B}}_{k}},
$$

with the diagonal energy matrix

$$
\begin{aligned}
\mathbb{E}_{\boldsymbol{k}} & \equiv\left(\mathbb{P}_{\boldsymbol{k}}^{\dagger}\right)^{-1} \mathbb{H}_{\boldsymbol{k}} \mathbb{P}_{\boldsymbol{k}}^{-1} \\
& =\operatorname{diag}\left(\varepsilon_{\boldsymbol{k}, 1}, \ldots, \varepsilon_{\boldsymbol{k}, N}, \varepsilon_{-\boldsymbol{k}, 1}, \ldots, \varepsilon_{-\boldsymbol{k}, N}\right) .
\end{aligned}
$$

The paraunitarity of $\mathbb{P}_{\boldsymbol{k}}$ is expressed by

$$
\mathbb{U}=\mathbb{P}_{\boldsymbol{k}}^{\dagger} \mathbb{U} \mathbb{P}_{\boldsymbol{k}}=\operatorname{diag}\left(1_{N},-1_{N}\right) .
$$

Here $\mathbb{U}$ is the bosonic metric, necessary to preserve bosonic commutation rules. In addition, $1_{N}$ is the $N \times N$ unit matrix. Note the relation

$$
\mathbb{P}_{\boldsymbol{k}}^{-1}=\mathbb{U} \mathbb{P}_{\boldsymbol{k}}^{\dagger} \mathbb{U}
$$

The above diagonalization procedure follows Ref. [154].

Below we need the expressions

$$
\hat{\boldsymbol{A}}_{\boldsymbol{k}}=\mathbb{P}_{\boldsymbol{k}}^{-1} \hat{\boldsymbol{B}}_{\boldsymbol{k}}=\left(\begin{array}{cc}
\mathbb{X}_{\boldsymbol{k}}^{\mathrm{T}} & \mathbb{Y}_{-\boldsymbol{k}}^{\dagger} \\
\mathbb{Y}_{\boldsymbol{k}}^{\mathrm{T}} & \mathbb{X}_{-\boldsymbol{k}}^{\dagger}
\end{array}\right) \hat{\boldsymbol{B}}_{\boldsymbol{k}},
$$

or elementwise

$$
\begin{aligned}
\hat{a}_{\boldsymbol{k}, \alpha} & =\sum_{\lambda=1}^{N}\left[\left(\mathbb{X}_{\boldsymbol{k}}\right)_{\lambda \alpha} \hat{b}_{\boldsymbol{k}, \lambda}+\left(\mathbb{Y}_{-\boldsymbol{k}}^{*}\right)_{\lambda \alpha} \hat{b}_{-\boldsymbol{k}, \lambda}^{\dagger}\right], \\
\hat{a}_{-\boldsymbol{k}, \alpha}^{\dagger} & =\sum_{\lambda=1}^{N}\left[\left(\mathbb{X}_{-\boldsymbol{k}}^{*}\right)_{\lambda \alpha} \hat{b}_{-\boldsymbol{k}, \lambda}^{\dagger}+\left(\mathbb{Y}_{\boldsymbol{k}}\right)_{\lambda \alpha} \hat{b}_{\boldsymbol{k}, \lambda}\right] .
\end{aligned}
$$

To obtain $\mathbb{P}_{\boldsymbol{k}}$ 's submatrices $\mathbb{X}_{\boldsymbol{k}}$ and $\mathbb{Y}_{\boldsymbol{k}}$ we invoke the following numerical recipe. We unitarily diagonalize $\mathbb{U H}_{\boldsymbol{k}}$ and then paranormalize the resulting eigenvectors, i.e., the columns of $\mathbb{P}_{\boldsymbol{k}}^{-1}$, by enforcing Eq. (A29). With $\mathbb{P}_{\boldsymbol{k}}^{-1}$ at hand, Eq. (A31) is used to read off $\mathbb{X}_{k}$ and $\mathbb{Y}_{\boldsymbol{k}}$.

\section{b. Anharmonic (cubic) theory}

Before turning to $\hat{H}_{3}$, we note that spin-wave theory is an expansion about a (meta)stable state, that is, about a (local or global) energy minimum. Consequently, there are no forces that drive the system into a different state. In the second-quantized language this means that there is no spontaneous creation (decay) of a single particle out of (into) vacuum. Mathematically, this is expressed by

$$
\begin{aligned}
0=\hat{H}_{1}= & \frac{S \sqrt{S}}{2} \sum_{\boldsymbol{r}, \boldsymbol{r}^{\prime}} \sum_{\boldsymbol{r}_{\mu}, \boldsymbol{r}_{v}}\left(\hat{a}_{\boldsymbol{r}+\boldsymbol{r}_{\mu}} \mathcal{I}_{\boldsymbol{r}+\boldsymbol{r}_{\mu}, \boldsymbol{r}^{\prime}+\boldsymbol{r}_{v}}^{-z}+\hat{a}_{\boldsymbol{r}+\boldsymbol{r}_{\mu}}^{\dagger} \mathcal{I}_{\boldsymbol{r}+\boldsymbol{r}_{\mu}, \boldsymbol{r}^{\prime}+\boldsymbol{r}_{v}}^{+z}+\hat{a}_{\boldsymbol{r}^{\prime}+\boldsymbol{r}_{v}} \mathcal{I}_{\boldsymbol{r}+\boldsymbol{r}_{\mu}, \boldsymbol{r}^{\prime}+\boldsymbol{r}_{v}}^{z-}+\hat{a}_{\boldsymbol{r}^{\prime}+\boldsymbol{r}_{v}}^{\dagger} \mathcal{I}_{\boldsymbol{r}+\boldsymbol{r}_{\mu}, \boldsymbol{r}^{\prime}+\boldsymbol{r}_{v}}^{z+}\right) \\
& +\sqrt{S} \sum_{\boldsymbol{r}} \sum_{\boldsymbol{r}_{\mu}}\left(\hat{a}_{\boldsymbol{r}+\boldsymbol{r}_{\mu}} \boldsymbol{b} \cdot \boldsymbol{e}_{\mu}^{-}+\hat{a}_{\boldsymbol{r}+\boldsymbol{r}_{\mu}}^{\dagger} \boldsymbol{b} \cdot \boldsymbol{e}_{\mu}^{+}\right)
\end{aligned}
$$


This condition can be used to find the classical magnetic ground state. In our numerical setup, we directly minimize $\hat{H}_{0}$, which is an equivalent procedure. Numerically, the absence of $\hat{H}_{1}$ can be checked by the positivity and realness of the single-particle energies.

The full cubic Hamiltonian after HP transformation reads

$$
\begin{aligned}
\hat{H}_{3}= & -\frac{\sqrt{S}}{2} \sum_{\boldsymbol{r}, \boldsymbol{r}^{\prime}} \sum_{\boldsymbol{r}_{\mu}, \boldsymbol{r}_{v}}\left(\hat{a}_{\boldsymbol{r}+\boldsymbol{r}_{\mu}} \hat{a}_{\boldsymbol{r}^{\prime}+\boldsymbol{r}_{v}}^{\dagger} \hat{a}_{\boldsymbol{r}^{\prime}+\boldsymbol{r}_{v}} \mathcal{I}_{\boldsymbol{r}+\boldsymbol{r}_{\mu}, \boldsymbol{r}^{\prime}+\boldsymbol{r}_{v}}^{-z}+\hat{a}_{\boldsymbol{r}+\boldsymbol{r}_{\mu}}^{\dagger} \hat{a}_{\boldsymbol{r}^{\prime}+\boldsymbol{r}_{v}}^{\dagger} \hat{a}_{\boldsymbol{r}^{\prime}+\boldsymbol{r}_{v}} \mathcal{I}_{\boldsymbol{r}+\boldsymbol{r}_{\mu}, \boldsymbol{r}^{\prime}+\boldsymbol{r}_{v}}^{+z}\right. \\
& \left.+\hat{a}_{\boldsymbol{r}+\boldsymbol{r}_{\mu}}^{\dagger} \hat{a}_{\boldsymbol{r}+\boldsymbol{r}_{\mu}} \hat{a}_{\boldsymbol{r}^{\prime}+\boldsymbol{r}_{v}} \mathcal{I}_{\boldsymbol{r}+\boldsymbol{r}_{\mu}, \boldsymbol{r}^{\prime}+\boldsymbol{r}_{v}}^{z-}+\hat{a}_{\boldsymbol{r}+\boldsymbol{r}_{\mu}}^{\dagger} \hat{a}_{\boldsymbol{r}+\boldsymbol{r}_{\mu}} \hat{a}_{\boldsymbol{r}^{\prime}+\boldsymbol{r}_{v}}^{\dagger} \mathcal{I}_{\boldsymbol{r}+\boldsymbol{r}_{\mu}, \boldsymbol{r}^{\prime}+\boldsymbol{r}_{v}}^{z+}\right)-\frac{1}{4}\left[\frac { \sqrt { S } } { 2 } \sum _ { \boldsymbol { r } , \boldsymbol { r } ^ { \prime } } \sum _ { \boldsymbol { r } _ { \mu } , \boldsymbol { r } _ { v } } \left(\hat{a}_{\boldsymbol{r}+\boldsymbol{r}_{\mu}}^{\dagger} \hat{a}_{\boldsymbol{r}+\boldsymbol{r}_{\mu}} \hat{a}_{\boldsymbol{r}+\boldsymbol{r}_{\mu}} \mathcal{I}_{\boldsymbol{r}+\boldsymbol{r}_{\mu}, \boldsymbol{r}^{\prime}+\boldsymbol{r}_{v}}^{-z}\right.\right. \\
& \left.+\hat{a}_{\boldsymbol{r}+\boldsymbol{r}_{\mu}}^{\dagger} \hat{a}_{\boldsymbol{r}+\boldsymbol{r}_{\mu}}^{\dagger} \hat{a}_{\boldsymbol{r}+\boldsymbol{r}_{\mu}} \mathcal{I}_{\boldsymbol{r}+\boldsymbol{r}_{\mu}, \boldsymbol{r}^{\prime}+\boldsymbol{r}_{v}}^{++z} \hat{a}_{\boldsymbol{r}^{\prime}+\boldsymbol{r}_{v}}^{\dagger} \hat{a}_{\boldsymbol{r}^{\prime}+\boldsymbol{r}_{v}} \hat{a}_{\boldsymbol{r}^{\prime}+\boldsymbol{r}_{v}} \mathcal{I}_{\boldsymbol{r}+\boldsymbol{r}_{\mu}, \boldsymbol{r}^{\prime}+\boldsymbol{r}_{v}}^{z-}+\hat{a}_{\boldsymbol{r}^{\prime}+\boldsymbol{r}_{v}}^{\dagger} a_{\boldsymbol{r}^{\prime}+\boldsymbol{r}_{v}}^{\dagger} \hat{a}_{\boldsymbol{r}^{\prime}+\boldsymbol{r}_{v}} \mathcal{I}_{\boldsymbol{r}+\boldsymbol{r}_{\mu}, \boldsymbol{r}^{\prime}+\boldsymbol{r}_{v}}^{z+}\right) \\
& \left.+\frac{1}{\sqrt{S}} \sum_{\boldsymbol{r}} \sum_{\boldsymbol{r}_{\mu}}\left(\hat{a}_{\boldsymbol{r}+\boldsymbol{r}_{\mu}}^{\dagger} \hat{a}_{\boldsymbol{r}+\boldsymbol{r}_{\mu}} \hat{a}_{\boldsymbol{r}+\boldsymbol{r}_{\mu}} \boldsymbol{b} \cdot \boldsymbol{e}_{\mu}^{-}+\hat{a}_{\boldsymbol{r}+\boldsymbol{r}_{\mu}}^{\dagger} \hat{a}_{\boldsymbol{r}+\boldsymbol{r}_{\mu}}^{\dagger} \hat{a}_{\boldsymbol{r}+\boldsymbol{r}_{\mu}} \boldsymbol{b} \cdot \boldsymbol{e}_{\mu}^{+}\right)\right]
\end{aligned}
$$

The term with the $\frac{1}{4}$ factor in front has the same structure as $\hat{H}_{1}$ because within each term all bosonic operators belong to the same site. Consequently, if $\hat{H}_{1}$ vanishes, so does this part of $\hat{H}_{3}$. In the remaining part of $\hat{H}_{3}$, we move creators to the left of annihilators,

$$
\hat{H}_{3}=-\frac{\sqrt{S}}{2} \sum_{\boldsymbol{r}, \boldsymbol{r}^{\prime}} \sum_{\boldsymbol{r}_{\mu}, \boldsymbol{r}_{v}}\left(\hat{a}_{\boldsymbol{r}+\boldsymbol{r}_{\mu}}^{\dagger} \hat{a}_{\boldsymbol{r}^{\prime}+\boldsymbol{r}_{v}}^{\dagger} \hat{a}_{\boldsymbol{r}^{\prime}+\boldsymbol{r}_{v}} \mathcal{I}_{\boldsymbol{r}+\boldsymbol{r}_{\mu}, \boldsymbol{r}^{\prime}+\boldsymbol{r}_{v}}^{+z}+\hat{a}_{\boldsymbol{r}+\boldsymbol{r}_{\mu}}^{\dagger} \hat{a}_{\boldsymbol{r}^{\prime}+\boldsymbol{r}_{v}}^{\dagger} \hat{a}_{\boldsymbol{r}+\boldsymbol{r}_{\mu}} \mathcal{I}_{\boldsymbol{r}+\boldsymbol{r}_{\mu}, \boldsymbol{r}^{\prime}+\boldsymbol{r}_{v}}^{z+}+\text { H.c. }\right) .
$$

We used that no spin interacts with itself, i.e., $\mathbb{I}_{\boldsymbol{r}, \boldsymbol{r}}=0$.

After a Fourier transformation, Eq. (A35) assumes the form

$$
\hat{H}_{3}=-\frac{\sqrt{S}}{2 \sqrt{N_{\mathrm{u}}}} \sum_{\boldsymbol{k}, \boldsymbol{q}} \sum_{\mu, v}\left(\hat{a}_{\boldsymbol{k}, \mu}^{\dagger} \hat{a}_{\boldsymbol{q}, \nu}^{\dagger} \hat{a}_{\boldsymbol{k}+\boldsymbol{q}, v} \mathcal{I}_{\boldsymbol{k}, \mu \nu}^{+z}+\hat{a}_{\boldsymbol{k}, \mu}^{\dagger} \hat{a}_{\boldsymbol{q}, v}^{\dagger} \hat{a}_{\boldsymbol{k}+\boldsymbol{q}, \mu} \mathcal{I}_{-\boldsymbol{q}, \mu \nu}^{z+}+\text { H.c. }\right) .
$$

We simplified notation: The sum over $\boldsymbol{r}_{\mu}$ and $\boldsymbol{r}_{\nu}$ is denoted as a sum over $\mu$ and $\nu$. One can show that $\mathcal{I}_{-\boldsymbol{q}, \mu \nu}^{z+}=\left(\mathcal{I}_{-\boldsymbol{q}, \mu \nu}^{z+}\right)^{\mathrm{T}}=\mathcal{I}_{\boldsymbol{q}, \nu \mu}^{+z}$, using the general property $\mathbb{I}_{\boldsymbol{r}, \boldsymbol{r}^{\prime}}=\left(\mathbb{I}_{\boldsymbol{r}^{\prime}, \boldsymbol{r}}\right)^{\mathrm{T}}$ of magnetic interaction matrices. Finally, we find the following generic form of the cubic Hamiltonian in terms of HP bosons:

$$
\hat{H}_{3}=\frac{\sqrt{S}}{\sqrt{N_{\mathrm{u}}}} \sum_{\lambda, \mu, \nu} \sum_{\boldsymbol{k}, \boldsymbol{q}, \boldsymbol{p}}^{\boldsymbol{p}=\boldsymbol{k}+\boldsymbol{q}}\left(\frac{1}{2 ! 1 !} V_{\boldsymbol{k}, \boldsymbol{q} \leftarrow \boldsymbol{p}}^{\lambda \mu \leftarrow v} \hat{a}_{\boldsymbol{k}, \lambda}^{\dagger} \hat{a}_{\boldsymbol{q}, \mu}^{\dagger} \hat{a}_{\boldsymbol{p}, \nu}+\text { H.c. }\right) .
$$

The interaction vertex $V_{\boldsymbol{k}, \boldsymbol{q} \leftarrow \boldsymbol{p}}^{\lambda \mu \leftarrow \nu}=-\delta_{\mu \nu} \mathcal{I}_{\boldsymbol{k}, \lambda \mu}^{+z}-\delta_{\lambda \nu} \mathcal{I}_{\boldsymbol{q}, \mu \lambda}^{+z}$ is a complex number in general; its expression agrees with that derived in Ref. [155]. The factorial factors are written to stress the symmetric nature of $\hat{H}_{3}$, i.e., creators and annihilators can be permuted among themselves.

In terms of normal modes, Eqs. (A32a) and (A32b), the cubic Hamiltonian reads

$$
\begin{aligned}
\hat{H}_{3}= & \frac{\sqrt{S}}{2 \sqrt{N}} \sum_{\alpha, \beta, \gamma} \sum_{\boldsymbol{k}, \boldsymbol{q}, \boldsymbol{p}}^{\boldsymbol{p}=\boldsymbol{k}+\boldsymbol{q}}\left[V_{\boldsymbol{k}, \boldsymbol{q} \leftarrow \boldsymbol{p}}^{\alpha \beta \leftarrow \gamma} \hat{a}_{\boldsymbol{k}, \alpha}^{\dagger} \hat{a}_{\boldsymbol{q}, \beta}^{\dagger} \hat{a}_{\boldsymbol{p}, \gamma}+\left(V_{\boldsymbol{k}, \boldsymbol{q} \leftarrow \boldsymbol{p}}^{\alpha \beta \leftarrow \gamma}\right)^{*} \hat{a}_{\boldsymbol{p}, \gamma}^{\dagger} \hat{a}_{\boldsymbol{q}, \beta} \hat{a}_{\boldsymbol{k}, \alpha}\right] \\
= & \frac{\sqrt{S}}{2 \sqrt{N}} \sum_{\alpha, \beta, \gamma} \sum_{\lambda, \mu, \nu} \sum_{\boldsymbol{k}, \boldsymbol{q}, \boldsymbol{p}}^{\boldsymbol{p}=\boldsymbol{k}+\boldsymbol{q}}\left\{V_{\boldsymbol{k}, \boldsymbol{q} \leftarrow \boldsymbol{p}}^{\alpha \beta \leftarrow \gamma}\left[\left(\mathbb{X}_{\boldsymbol{k}}^{*}\right)_{\lambda \alpha} \hat{b}_{\boldsymbol{k}, \lambda}^{\dagger}+\left(\mathbb{Y}_{-\boldsymbol{k}}\right)_{\lambda \alpha} \hat{b}_{-\boldsymbol{k}, \lambda}\right]\left[\left(\mathbb{X}_{\boldsymbol{q}}^{*}\right)_{\mu \beta} \hat{b}_{\boldsymbol{q}, \mu}^{\dagger}+\left(\mathbb{Y}_{-\boldsymbol{q}}\right)_{\mu \beta} \hat{b}_{-\boldsymbol{q}, \mu}\right]\left[\left(\mathbb{X}_{\boldsymbol{p}}\right)_{\nu \gamma} \hat{b}_{\boldsymbol{p}, \nu}+\left(\mathbb{Y}_{-\boldsymbol{p}}^{*}\right)_{\nu \gamma} \hat{b}_{-\boldsymbol{p}, \nu}^{\dagger}\right]\right. \\
& \left.+\left(V_{\boldsymbol{k}, \boldsymbol{q} \leftarrow \boldsymbol{p}}^{\alpha \beta \leftarrow \gamma}\right)^{*}\left[\left(\mathbb{X}_{\boldsymbol{p}}^{*}\right)_{\nu \gamma} \hat{b}_{\boldsymbol{p}, \nu}^{\dagger}+\left(\mathbb{Y}_{-\boldsymbol{p}}\right)_{\nu \gamma} \hat{b}_{-\boldsymbol{p}, \nu}\right]\left[\left(\mathbb{X}_{\boldsymbol{q}}\right)_{\mu \beta} \hat{b}_{\boldsymbol{q}, \mu}+\left(\mathbb{Y}_{-\boldsymbol{q}}^{*}\right)_{\mu \beta} \hat{b}_{-\boldsymbol{q}, \mu}^{\dagger}\right]\left[\left(\mathbb{X}_{\boldsymbol{k}}\right)_{\lambda \alpha} \hat{b}_{\boldsymbol{k}, \lambda}+\left(\mathbb{Y}_{-\boldsymbol{k}}^{*}\right)_{\lambda \alpha} \hat{b}_{-\boldsymbol{k}, \lambda}^{\dagger}\right]\right\} .
\end{aligned}
$$

Upon expansion, one finds that the cubic Hamiltonian assumes the form $\hat{H}_{3}=\hat{H}_{3}^{\mathrm{d}}+\hat{H}_{3}^{\mathrm{s}}$, where the decay (d) part reads

$$
\begin{aligned}
\hat{H}_{3}^{\mathrm{d}}= & \frac{\sqrt{S}}{2 \sqrt{N}} \sum_{\alpha, \beta, \gamma} \sum_{\lambda, \mu, \nu} \sum_{\boldsymbol{k}, \boldsymbol{q}, \boldsymbol{p}}^{\boldsymbol{p}=\boldsymbol{k}+\boldsymbol{q}}\left[V_{\boldsymbol{k}, \boldsymbol{q} \leftarrow \boldsymbol{p}}^{\alpha \beta \leftarrow \gamma}\left(\mathbb{X}_{\boldsymbol{k}}^{*}\right)_{\lambda \alpha}\left(\mathbb{X}_{\boldsymbol{q}}^{*}\right)_{\mu \beta}\left(\mathbb{X}_{\boldsymbol{p}}\right)_{\nu \gamma} \hat{b}_{\boldsymbol{k}, \lambda}^{\dagger} \hat{b}_{\boldsymbol{q}, \mu}^{\dagger} \hat{b}_{\boldsymbol{p}, \nu}+\left(V_{\boldsymbol{k}, \boldsymbol{q} \leftarrow \boldsymbol{p}}^{\alpha \beta \leftarrow \gamma}\right)^{*}\left(\mathbb{Y}_{-\boldsymbol{k}}^{*}\right)_{\lambda \alpha}\left(\mathbb{Y}_{-\boldsymbol{q}}^{*}\right)_{\mu \beta}\left(\mathbb{Y}_{-\boldsymbol{p}}\right)_{\nu \gamma} \hat{b}_{-\boldsymbol{k}, \lambda}^{\dagger} \hat{b}_{-\boldsymbol{q}, \mu}^{\dagger} \hat{b}_{-\boldsymbol{p}, \nu}\right. \\
& +V_{\boldsymbol{k}, \boldsymbol{q} \leftarrow \boldsymbol{p}}^{\alpha \beta \leftarrow \gamma}\left(\mathbb{X}_{\boldsymbol{k}}^{*}\right)_{\lambda \alpha}\left(\mathbb{Y}_{-\boldsymbol{q}}\right)_{\mu \beta}\left(\mathbb{Y}_{-\boldsymbol{p}}^{*}\right)_{\nu \gamma} \hat{b}_{-\boldsymbol{p}, \nu}^{\dagger} \hat{b}_{\boldsymbol{k}, \lambda}^{\dagger} \hat{b}_{-\boldsymbol{q}, \mu}+\left(V_{\boldsymbol{k}, \boldsymbol{q} \leftarrow \boldsymbol{p}}^{\alpha \beta \leftarrow \gamma}\right)^{*}\left(\mathbb{Y}_{-\boldsymbol{k}}^{*}\right)_{\lambda \alpha}\left(\mathbb{X}_{\boldsymbol{q}}\right)_{\mu \beta}\left(\mathbb{X}_{\boldsymbol{p}}^{*}\right)_{\nu \gamma} \hat{b}_{\boldsymbol{p}, \nu}^{\dagger} \hat{b}_{-\boldsymbol{k}, \lambda}^{\dagger} \hat{b}_{\boldsymbol{q}, \mu} \\
& \left.+V_{\boldsymbol{k}, \boldsymbol{q} \leftarrow \boldsymbol{p}}^{\alpha \beta \leftarrow \gamma}\left(\mathbb{Y}_{-\boldsymbol{k}}\right)_{\lambda \alpha}\left(\mathbb{X}_{\boldsymbol{q}}^{*}\right)_{\mu \beta}\left(\mathbb{Y}_{-\boldsymbol{p}}^{*}\right)_{\nu \gamma} \hat{b}_{\boldsymbol{q}, \mu}^{\dagger} \hat{b}_{-\boldsymbol{p}, \nu}^{\dagger} \hat{b}_{-\boldsymbol{k}, \lambda}\left(V_{\boldsymbol{k}, \boldsymbol{q} \leftarrow \boldsymbol{p}}^{\alpha \beta \leftarrow \gamma}\right)^{*}\left(\mathbb{X}_{\boldsymbol{k}}\right)_{\lambda \alpha}\left(\mathbb{Y}_{-\boldsymbol{q}}^{*}\right)_{\mu \beta}\left(\mathbb{X}_{\boldsymbol{p}}^{*}\right)_{\nu \gamma} \hat{b}_{-\boldsymbol{q}, \mu}^{\dagger} \hat{b}_{\boldsymbol{p}, \nu}^{\dagger} \hat{b}_{\boldsymbol{k}, \lambda}+\mathrm{H} . \mathrm{c} .\right]
\end{aligned}
$$


and the source (s) part

$$
\begin{aligned}
\hat{H}_{3}^{\mathrm{s}}= & \frac{\sqrt{S}}{2 \sqrt{N}} \sum_{\alpha, \beta, \gamma} \sum_{\lambda, \mu, \nu} \sum_{\boldsymbol{k}, \boldsymbol{q}, \boldsymbol{p}}^{\boldsymbol{p}=\boldsymbol{k}+\boldsymbol{q}}\left[V_{\boldsymbol{k}, \boldsymbol{q} \leftarrow \boldsymbol{p}}^{\alpha \beta \leftarrow \gamma}\left(\mathbb{X}_{\boldsymbol{k}}^{*}\right)_{\lambda \alpha}\left(\mathbb{X}_{\boldsymbol{q}}^{*}\right)_{\mu \beta}\left(\mathbb{Y}_{-\boldsymbol{p}}^{*}\right)_{\nu \gamma} \hat{b}_{\boldsymbol{k}, \lambda}^{\dagger} \hat{b}_{\boldsymbol{q}, \mu}^{\dagger} \hat{b}_{-\boldsymbol{p}, \nu}^{\dagger}\right. \\
& \left.+\left(V_{\boldsymbol{k}, \boldsymbol{q} \leftarrow \boldsymbol{p}}^{\alpha \beta \leftarrow \gamma}\right)^{*}\left(\mathbb{X}_{\boldsymbol{p}}^{*}\right)_{\nu \gamma}\left(\mathbb{Y}_{-\boldsymbol{q}}^{*}\right)_{\mu \beta}\left(\mathbb{Y}_{-\boldsymbol{k}}^{*}\right)_{\lambda \alpha} \hat{b}_{\boldsymbol{p}, \nu}^{\dagger} \hat{b}_{-\boldsymbol{q}, \mu}^{\dagger} \hat{b}_{-\boldsymbol{k}, \lambda}^{\dagger}+\text { H.c. }\right] .
\end{aligned}
$$

We now reverse the sign of the momenta in every second term to obtain

$$
\begin{aligned}
\hat{H}_{3}^{\mathrm{d}}= & \frac{\sqrt{S}}{2 \sqrt{N}} \sum_{\alpha, \beta, \gamma} \sum_{\lambda, \mu, \nu} \sum_{\boldsymbol{k}, \boldsymbol{q}, \boldsymbol{p}}^{\boldsymbol{p}=\boldsymbol{k}+\boldsymbol{q}}\left\{\left[V_{\boldsymbol{k}, \boldsymbol{q} \leftarrow \boldsymbol{p}}^{\alpha \beta \leftarrow \gamma}\left(\mathbb{X}_{\boldsymbol{k}}^{*}\right)_{\lambda \alpha}\left(\mathbb{X}_{\boldsymbol{q}}^{*}\right)_{\mu \beta}\left(\mathbb{X}_{\boldsymbol{p}}\right)_{\nu \gamma}+\left(V_{-\boldsymbol{k},-\boldsymbol{q} \leftarrow-\boldsymbol{p}}^{\alpha \beta \leftarrow \gamma}\right)^{*}\left(\mathbb{Y}_{\boldsymbol{k}}^{*}\right)_{\lambda \alpha}\left(\mathbb{Y}_{\boldsymbol{q}}^{*}\right)_{\mu \beta}\left(\mathbb{Y}_{\boldsymbol{p}}\right)_{\nu \gamma}\right] \hat{b}_{\boldsymbol{k}, \lambda}^{\dagger} \hat{b}_{\boldsymbol{q}, \mu}^{\dagger} \hat{b}_{\boldsymbol{p}, \nu}\right. \\
& +\left[V_{\boldsymbol{k}, \boldsymbol{q} \leftarrow \boldsymbol{p}}^{\alpha \beta \leftarrow \gamma}\left(\mathbb{X}_{\boldsymbol{k}}^{*}\right)_{\lambda \alpha}\left(\mathbb{Y}_{-\boldsymbol{q}}\right)_{\mu \beta}\left(\mathbb{Y}_{-\boldsymbol{p}}^{*}\right)_{\nu \gamma}+\left(V_{-\boldsymbol{k},-\boldsymbol{q} \leftarrow-\boldsymbol{p}}^{\alpha \beta \leftarrow \gamma}\right)^{*}\left(\mathbb{Y}_{\boldsymbol{k}}^{*}\right)_{\lambda \alpha}\left(\mathbb{X}_{-\boldsymbol{q}}\right)_{\mu \beta}\left(\mathbb{X}_{-\boldsymbol{p}}^{*}\right)_{\nu \gamma}\right] \hat{b}_{-\boldsymbol{p}, \nu}^{\dagger} \hat{b}_{\boldsymbol{k}, \lambda}^{\dagger} \hat{b}_{-\boldsymbol{q}, \mu} \\
& \left.+\left[V_{\boldsymbol{k}, \boldsymbol{q} \leftarrow \boldsymbol{p}}^{\alpha \beta \leftarrow \gamma}\left(\mathbb{Y}_{-\boldsymbol{k}}\right)_{\lambda \alpha}\left(\mathbb{X}_{\boldsymbol{q}}^{*}\right)_{\mu \beta}\left(\mathbb{Y}_{-\boldsymbol{p}}^{*}\right)_{\nu \gamma}+\left(V_{-\boldsymbol{k}, \boldsymbol{q} \leftarrow-\boldsymbol{p}}^{\alpha \beta \leftarrow \gamma}\right)^{*}\left(\mathbb{X}_{-\boldsymbol{k}}\right)_{\lambda \alpha}\left(\mathbb{Y}_{\boldsymbol{q}}^{*}\right)_{\mu \beta}\left(\mathbb{X}_{-\boldsymbol{p}}^{*}\right)_{\nu \gamma}\right] \hat{b}_{\boldsymbol{q}, \mu}^{\dagger} \hat{b}_{-\boldsymbol{p}, \nu}^{\dagger} \hat{b}_{-\boldsymbol{k}, \lambda}+\text { H.c. }\right\}
\end{aligned}
$$

and

$$
\hat{H}_{3}^{\mathrm{s}}=\frac{\sqrt{S}}{2 \sqrt{N}} \sum_{\alpha, \beta, \gamma} \sum_{\lambda, \mu, \nu} \sum_{\boldsymbol{k}, \boldsymbol{q}, \boldsymbol{p}}^{\boldsymbol{p}=\boldsymbol{k}+\boldsymbol{q}}\left\{\left[V_{\boldsymbol{k}, \boldsymbol{q} \leftarrow \boldsymbol{p}}^{\alpha \beta \leftarrow \gamma}\left(\mathbb{X}_{\boldsymbol{k}}^{*}\right)_{\lambda \alpha}\left(\mathbb{X}_{\boldsymbol{q}}^{*}\right)_{\mu \beta}\left(\mathbb{Y}_{-\boldsymbol{p}}^{*}\right)_{\nu \gamma}+\left(V_{-\boldsymbol{k}, \boldsymbol{q} \leftarrow-\boldsymbol{p}}^{\alpha \beta \leftarrow \gamma}\right)^{*}\left(\mathbb{X}_{-\boldsymbol{p}}^{*}\right)_{\nu \gamma}\left(\mathbb{Y}_{\boldsymbol{q}}^{*}\right)_{\mu \beta}\left(\mathbb{Y}_{\boldsymbol{k}}^{*}\right)_{\lambda \alpha}\right] \hat{b}_{\boldsymbol{k}, \lambda}^{\dagger} \hat{b}_{\boldsymbol{q}, \mu}^{\dagger} \hat{b}_{-\boldsymbol{p}, \nu}^{\dagger}+\mathrm{H} . \mathrm{c} .\right\}
$$

Finally, for symmetrization, we relabel momenta and band indices in the second and third lines of Eq. (A41). Using $v \rightarrow \lambda \rightarrow$ $\mu \rightarrow v$ and $-\boldsymbol{p} \rightarrow \boldsymbol{k} \rightarrow \boldsymbol{q} \rightarrow-\boldsymbol{p}$ in the second line and $\mu \rightarrow \lambda \rightarrow v \rightarrow \mu$ and $\boldsymbol{q} \rightarrow \boldsymbol{k} \rightarrow-\boldsymbol{p} \rightarrow \boldsymbol{q}$ in the third line, we arrive at

$$
\hat{H}_{3}^{\mathrm{d}}=\frac{\sqrt{S}}{\sqrt{N}} \sum_{\lambda, \mu, \nu} \sum_{\boldsymbol{k}, \boldsymbol{q}, \boldsymbol{p}}^{\boldsymbol{p}=\boldsymbol{k}+\boldsymbol{q}}\left(\frac{1}{2 ! 1 !} \mathcal{V}_{\boldsymbol{k}, \boldsymbol{q} \leftarrow \boldsymbol{p}}^{\lambda \mu \leftarrow v} \hat{b}_{\boldsymbol{k}, \lambda}^{\dagger} \hat{b}_{\boldsymbol{q}, \mu}^{\dagger} \hat{b}_{\boldsymbol{p}, v}+\text { H.c. }\right)
$$

with the decay vertex

$$
\begin{aligned}
\mathcal{V}_{\boldsymbol{k}, \boldsymbol{q} \leftarrow \boldsymbol{p}}^{\lambda \mu \leftarrow \nu}= & \sum_{\alpha, \beta, \gamma}\left[V_{\boldsymbol{k}, \boldsymbol{q} \leftarrow \boldsymbol{p}}^{\alpha \beta \leftarrow \gamma}\left(\mathbb{X}_{\boldsymbol{k}}^{*}\right)_{\lambda \alpha}\left(\mathbb{X}_{\boldsymbol{q}}^{*}\right)_{\mu \beta}\left(\mathbb{X}_{\boldsymbol{p}}\right)_{\nu \gamma}+\left(V_{-\boldsymbol{k},-\boldsymbol{q} \leftarrow-\boldsymbol{p}}^{\alpha \beta \leftarrow \gamma}\right)^{*}\left(\mathbb{Y}_{\boldsymbol{k}}^{*}\right)_{\lambda \alpha}\left(\mathbb{Y}_{\boldsymbol{q}}^{*}\right)_{\mu \beta}\left(\mathbb{Y}_{\boldsymbol{p}}\right)_{\nu \gamma}+V_{\boldsymbol{q},-\boldsymbol{p} \leftarrow-\boldsymbol{k}}^{\alpha \beta \leftarrow \gamma}\left(\mathbb{X}_{\boldsymbol{q}}^{*}\right)_{\mu \alpha}\left(\mathbb{Y}_{\boldsymbol{p}}\right)_{\nu \beta}\left(\mathbb{Y}_{\boldsymbol{k}}^{*}\right)_{\lambda \gamma}\right. \\
& \left.+\left(V_{-\boldsymbol{q}, \boldsymbol{p} \leftarrow \boldsymbol{k}}^{\alpha \beta \leftarrow \gamma}\right)\left(\mathbb{Y}_{\boldsymbol{q}}^{*}\right)_{\mu \alpha}\left(\mathbb{X}_{\boldsymbol{p}}\right)_{\nu \beta}\left(\mathbb{X}_{\boldsymbol{k}}^{*}\right)_{\lambda \gamma}+V_{-\boldsymbol{p}, \boldsymbol{k} \leftarrow-\boldsymbol{q}}^{\alpha \beta \leftarrow \gamma}\left(\mathbb{Y}_{\boldsymbol{p}}\right)_{\nu \alpha}\left(\mathbb{X}_{\boldsymbol{k}}^{*}\right)_{\lambda \beta}\left(\mathbb{Y}_{\boldsymbol{q}}^{*}\right)_{\mu \gamma}+\left(V_{\boldsymbol{p},-\boldsymbol{k} \leftarrow \boldsymbol{q}}^{\alpha \beta \leftarrow \gamma}\right)^{*}\left(\mathbb{X}_{\boldsymbol{p}}\right)_{\nu \alpha}\left(\mathbb{Y}_{\boldsymbol{k}}^{*}\right)_{\lambda \beta}\left(\mathbb{X}_{\boldsymbol{q}}^{*}\right)_{\mu \gamma}\right] .
\end{aligned}
$$

Similarly, in Eq. (A42), we add the summand another two times, divide by 3, and cyclically permute momenta and band indices. We arrive at

$$
\hat{H}_{3}^{\mathrm{s}}=\frac{\sqrt{S}}{\sqrt{N}} \sum_{\lambda, \mu, \nu} \sum_{\boldsymbol{k}, \boldsymbol{q}, \boldsymbol{p}}^{-\boldsymbol{p}=\boldsymbol{k}+\boldsymbol{q}}\left(\frac{1}{3 ! 0 !} \boldsymbol{W}_{\boldsymbol{k}, \boldsymbol{q}, \boldsymbol{p}}^{\lambda \mu \nu} \hat{b}_{\boldsymbol{k}, \lambda}^{\dagger} \hat{b}_{\boldsymbol{q}, \mu}^{\dagger} \hat{b}_{\boldsymbol{p}, \nu}^{\dagger}+\text { H.c. }\right)
$$

with the source vertex

$$
\begin{aligned}
\boldsymbol{W}_{\boldsymbol{k}, \boldsymbol{q}, \boldsymbol{p}}^{\lambda \mu \nu}= & \sum_{\alpha, \beta, \gamma}\left[V_{\boldsymbol{k}, \boldsymbol{q} \leftarrow \boldsymbol{p}}^{\alpha \beta \leftarrow \gamma}\left(\mathbb{X}_{\boldsymbol{k}}^{*}\right)_{\lambda \alpha}\left(\mathbb{X}_{\boldsymbol{q}}^{*}\right)_{\mu \beta}\left(\mathbb{Y}_{\boldsymbol{p}}^{*}\right)_{\nu \gamma}+\left(V_{-\boldsymbol{k},-\boldsymbol{q} \leftarrow-\boldsymbol{p}}^{\alpha \beta \leftarrow \gamma}\right)^{*}\left(\mathbb{Y}_{\boldsymbol{k}}^{*}\right)_{\lambda \alpha}\left(\mathbb{Y}_{\boldsymbol{q}}^{*}\right)_{\mu \beta}\left(\mathbb{X}_{\boldsymbol{p}}^{*}\right)_{\nu \gamma}+V_{\boldsymbol{q}, \boldsymbol{p} \leftarrow \boldsymbol{k}}^{\alpha \beta \leftarrow \gamma}\left(\mathbb{X}_{\boldsymbol{q}}^{*}\right)_{\mu \alpha}\left(\mathbb{X}_{\boldsymbol{p}}^{*}\right)_{\nu \beta}\left(\mathbb{Y}_{\boldsymbol{k}}^{*}\right)_{\lambda \gamma}\right. \\
& \left.+\left(V_{-\boldsymbol{q},-\boldsymbol{p} \leftarrow-\boldsymbol{k}}^{\alpha \beta \leftarrow \gamma}\right)^{*}\left(\mathbb{Y}_{\boldsymbol{q}}^{*}\right)_{\mu \alpha}\left(\mathbb{Y}_{\boldsymbol{p}}^{*}\right)_{\nu \beta}\left(\mathbb{X}_{\boldsymbol{k}}^{*}\right)_{\lambda \gamma}+V_{\boldsymbol{p}, \boldsymbol{k} \leftarrow \boldsymbol{q}}^{\alpha \beta \leftarrow \gamma}\left(\mathbb{X}_{\boldsymbol{p}}^{*}\right)_{\nu \alpha}\left(\mathbb{X}_{\boldsymbol{k}}^{*}\right)_{\lambda \beta}\left(\mathbb{Y}_{\boldsymbol{q}}^{*}\right)_{\mu \gamma}+\left(V_{-\boldsymbol{p},-\boldsymbol{k} \leftarrow-\boldsymbol{q}}^{\alpha \beta \leftarrow \gamma}\right)^{*}\left(\mathbb{Y}_{\boldsymbol{p}}^{*}\right)_{\nu \alpha}\left(\mathbb{Y}_{\boldsymbol{k}}^{*}\right)_{\lambda \beta}\left(\mathbb{X}_{\boldsymbol{q}}^{*}\right)_{\mu \gamma}\right]
\end{aligned}
$$

Again, we explicitly denoted the factorial factors, arising from symmetrization under permutation of creators (or annihilators) among themselves.

\section{Many-body perturbation theory up to order $1 / S$}

Up to second-order many-body perturbation theory, the normal one-magnon temperature Green's function $G_{k, v \nu^{\prime}}(\tau)=$ $-\left\langle\mathcal{T}_{\tau} b_{k, v}(\tau) b_{k, v^{\prime}}^{\dagger}\right\rangle$ reads [156]

$$
G_{\boldsymbol{k}, v v^{\prime}}(\tau) \approx G_{\boldsymbol{k}, v \mathcal{v}^{\prime}}^{(0)}(\tau)+\frac{1}{\hbar} \int_{0}^{\hbar \beta} d \tau_{1}\left\langle\mathcal{T}_{\tau} \hat{H}_{4}\left(\tau_{1}\right) \hat{b}_{\boldsymbol{k}, v}(\tau) \hat{b}_{\boldsymbol{k}, v^{\prime}}^{\dagger}\right\rangle_{(0)}^{\mathrm{con}}-\frac{1}{2 ! \hbar^{2}} \int_{0}^{\hbar \beta} d \tau_{1} \int_{0}^{\hbar \beta} d \tau_{2}\left\langle\mathcal{T}_{\tau} \hat{H}_{3}\left(\tau_{1}\right) \hat{H}_{3}\left(\tau_{2}\right) \hat{b}_{\boldsymbol{k}, v}(\tau) \hat{b}_{\boldsymbol{k}, v^{\prime}}^{\dagger}\right\rangle(0)
$$

with $G_{\boldsymbol{k}, v v^{\prime}}^{(0)}(\tau)$ the bare propagator. The $\tau$ 's denote imaginary time, $\mathcal{T}_{\tau}$ is the "time"-ordering operator, the subscript $(0)$ denotes averaging with respect to $\hat{H}_{2}$, and "con" refers to connected diagrams; $\beta=\left(k_{\mathrm{B}} T\right)^{-1}$, where $k_{\mathrm{B}}$ is Boltzmann's constant and $T$ temperature. Both the first-order and the second-order term are $1 / S$ corrections to $G_{\boldsymbol{k}, v v^{\prime}}^{(0)}(\tau)$. Note that a second-order term 

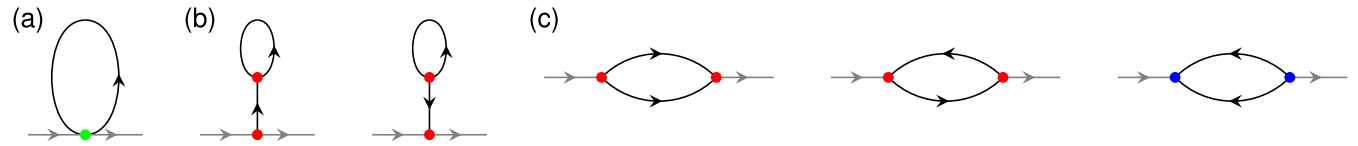

FIG. 7. Feynman diagrams of order- $1 / S$ self-energies. Gray (Black) lines denote external (internal) legs and green/red/blue circles denote interaction vertices. The green circle is the two-in-two-out four-magnon vertex (not given) and the red/blue circles are the decay $(\mathcal{V})$ and source $(\mathcal{W})$ three-magnon vertices (or their conjugates) given in Eqs. (A44) and (A46), respectively. (a) First-order Hartree-Fock corrections due to $\hat{H}_{4}$ that yield a static real self-energy. (b) Second-order tadpole diagrams due to $\hat{H}_{3}$ that also yield a static real self-energy. (c) Second-order bubble diagrams due to $\hat{H}_{3}$ that yield a dynamic self-energy. However, only the first two diagrams contribute to the imaginary part of the self-energy.

in $\hat{H}_{4}$ would yield a $1 / S^{2}$ correction and is therefore dropped. [Even for $S \leqslant 1$, spin-wave theory is surprisingly reliable and higher-order terms may be dropped because the expansion in Eq. (A17) is also effectively an expansion in the magnon number $\left\langle a^{\dagger} a\right\rangle$, which is small at low temperatures.]

By virtue of Wick's theorem, one shows that the first-order $\hat{H}_{4}$ term gives rise to frequency-independent Hartree-Fock corrections [cf. Fig. 7(a)] that cause a real self-energy [156,157]. Similarly, the second-order $\hat{H}_{3}$ term gives rise to frequencyindependent tadpole diagrams [cf. Fig. 7(b)] that also contribute a real self-energy [157]. Being interested in magnon damping, which is associated with the imaginary part of the self-energy, we drop all frequency-independent $1 / S$ corrections. Out of the remaining bubble diagrams [cf. Fig. 7(c)], which also derive from $\hat{H}_{3}$, only the first two yield complex diagonal normal self-energies $\left(v=v^{\prime}\right)$ :

$$
\Sigma_{\boldsymbol{k}, v}(\varepsilon)=\frac{1}{N_{\mathrm{u}}} \sum_{\boldsymbol{q}} \sum_{\lambda, \mu}\left(\frac{1}{2}\left|\mathcal{V}_{\boldsymbol{q}, \boldsymbol{k}-\boldsymbol{q} \leftarrow \boldsymbol{k}}^{\lambda \mu \leftarrow v}\right|^{2} \frac{\rho\left(\varepsilon_{\boldsymbol{q}, \lambda}\right)+\rho\left(\varepsilon_{\boldsymbol{k}-\boldsymbol{q}, \mu}\right)+1}{\varepsilon+i 0^{+}-\varepsilon_{\boldsymbol{q}, \lambda}-\varepsilon_{\boldsymbol{k}-\boldsymbol{q}, \mu}}+\left|\mathcal{V}_{\boldsymbol{k}, \boldsymbol{q} \leftarrow \boldsymbol{k}+\boldsymbol{q}}^{v \mu \leftarrow \lambda}\right|^{2} \frac{\rho\left(\varepsilon_{\boldsymbol{q}, \mu}\right)-\rho\left(\varepsilon_{\boldsymbol{k}+\boldsymbol{q}, \lambda}\right)}{\varepsilon+i 0^{+}+\varepsilon_{\boldsymbol{q}, \mu}-\varepsilon_{\boldsymbol{k}+\boldsymbol{q}, \lambda}}\right) .
$$

Here $\rho(x)=\left(e^{\beta x}-1\right)^{-1}$ is the Planck distribution. Note that the source vertex $\boldsymbol{W}_{\boldsymbol{k}, \boldsymbol{q}, \boldsymbol{p}}^{\lambda \mu \nu}$ does not enter Eq. (A48) because the source-sink bubble [right diagram of Fig. 7(c)] is off-resonance and we dropped it. Within the on-shell approximation $\varepsilon \rightarrow$ $\varepsilon_{\boldsymbol{k}, v}$, the magnon damping rate is given by $\Gamma_{k, v} \equiv-\operatorname{Im}\left[\Sigma_{\boldsymbol{k}, v}\left(\varepsilon_{\boldsymbol{k}, v}\right)\right]$. Ignoring the real part of the self-energy, the normal onemagnon (retarded) Green's function reads $G_{k, v}^{-1}(\varepsilon) \approx \varepsilon-\varepsilon_{k, v}+i \Gamma_{k, v}$, from which we obtain the spectral function $A_{k, v}(\varepsilon) \approx$ $-\operatorname{Im} G_{k, v}(\varepsilon) / \pi$ of band $v$. It follows from the Sokhotski-Plemelj theorem that the temperature-dependent damping reads

$$
\begin{aligned}
\Gamma_{\boldsymbol{k}, v}(T)= & \frac{\pi}{2 N_{\mathrm{u}}} \sum_{\boldsymbol{q}} \sum_{\lambda, \mu}\left|\mathcal{V}_{\boldsymbol{q}, \boldsymbol{k}-\boldsymbol{q} \leftarrow \boldsymbol{k}}^{\lambda \mu \leftarrow v}\right|^{2} \delta\left(\varepsilon_{\boldsymbol{k}, \nu}-\varepsilon_{\boldsymbol{q}, \lambda}-\varepsilon_{\boldsymbol{k}-\boldsymbol{q}, \mu}\right)\left[\rho\left(\varepsilon_{\boldsymbol{q}, \lambda}, T\right)+\rho\left(\varepsilon_{\boldsymbol{k}-\boldsymbol{q}, \mu}, T\right)+1\right] \\
& +\frac{\pi}{N_{\mathrm{u}}} \sum_{\boldsymbol{q}} \sum_{\lambda, \mu}\left|\mathcal{V}_{\boldsymbol{k}, \boldsymbol{q} \leftarrow \boldsymbol{k}+\boldsymbol{q}}^{\nu \mu \leftarrow \lambda}\right|^{2} \delta\left(\varepsilon_{\boldsymbol{k}, \nu}+\varepsilon_{\boldsymbol{q}, \mu}-\varepsilon_{\boldsymbol{k}+\boldsymbol{q}, \lambda}\right)\left[\rho\left(\varepsilon_{\boldsymbol{q}, \mu}, T\right)-\rho\left(\varepsilon_{\boldsymbol{k}+\boldsymbol{q}, \lambda}, T\right)\right] .
\end{aligned}
$$

The first line in Eq. (A49) comprises magnon decay. The spontaneous damping $\Gamma_{k, v}^{\text {spon }}$ in Eq. (2) is obtained by taking the limit $T \rightarrow 0$. The second line in Eq. (A49) is a two-magnon collision. It takes finite temperature to have thermal magnons available for collisions, which is why this term is frozen out as $T \rightarrow 0$.

\section{APPENDIX B: ADDITIONAL RESULTS}

\section{Extended Fig. 2}

Figure 8 extends Fig. 2. In Figs. 8(a)-8(g) the pronounced shift of the flat modes due to the Zeeman energy can be noticed. The two-magnon DOS, shown in Figs. 8(h)-8(n), inherits this field dependence, as revealed by the field-tunable sharp yellow features. The overlap of the single-particle bands with regions of a large two-magnon DOS leads to strong quasiparticle decay and lifetime band broadening in the spectral function [Figs. 8(o)-8(u)]. Several instances are marked by red arrows.

\section{Analysis of 1000 decay channels at $k=0$, involving the ten lowest magnon modes}

In the main text, we presented a decay channel analysis of the $\mathrm{ABC}$ modes. Here we extend this analysis to the lowest ten magnon bands, resulting in 1000 possible decay channels. In
Fig. 9 we use a data representation similar to that in Fig. 4 (histograms viewed from above and square color and size denoting the same information).

Two additional conclusions can be drawn.

First, note that the symbol $\times$ in Fig. 9 marks forbidden decay channels. (Numerically, we found that they have amplitudes less than $10^{-22} \mathrm{meV}^{2}$, which is negligible compared to other weak channels with amplitudes of the order of $10^{-4} \mathrm{meV}^{2}$.) Thus, decay channels with identical decay products are inactive for the G, A, T, C, v $=9$, and $\mathrm{P}$ modes. We conclude that these modes cannot decay into a single flat mode at half their energy. Note that this result is only correct for $\boldsymbol{k}=\mathbf{0}$ excitations. (We numerically verified that all decay channels are possible for $\boldsymbol{k} \neq \mathbf{0}$ excitations.)

Second, the decay efficiencies of polygon modes $[v=1$, 2, 4, 7, and 10 for the G, E, T, Q, and P mode in Figs. 9(a), 9(b), 9(d), 9(g), and 9(j), respectively] are overall larger than those of the ABC modes. Moreover, we find particularly large 


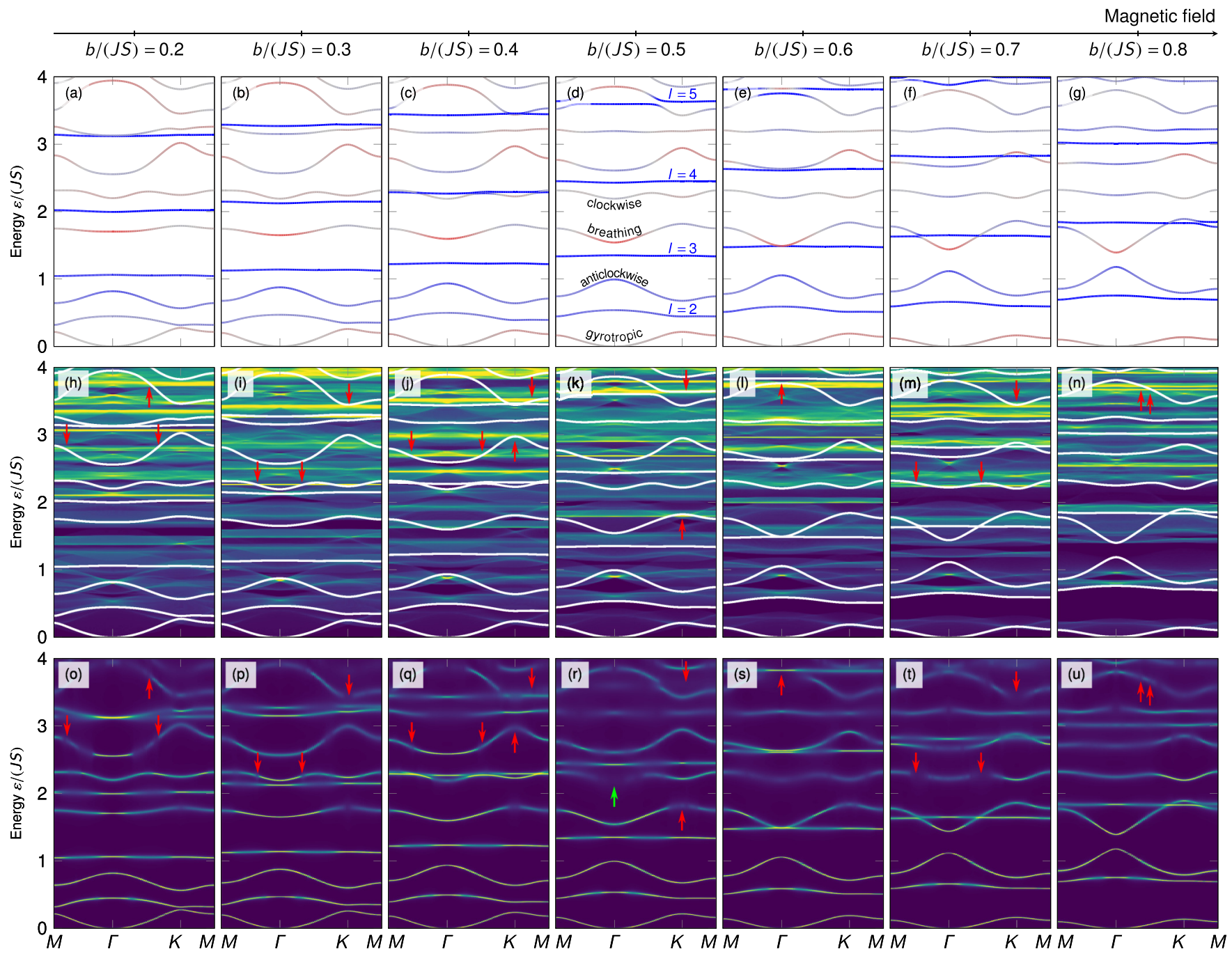

FIG. 8. Same as Fig. 2 but for additional magnetic field values.

$C^{\lambda \mu \leftarrow v}$ for decays that conserve $l$, e.g.,

$$
\begin{array}{rll}
10 \rightarrow(2,4), & l=5=2+3 & \text { [Fig. 9(j), P mode] }, \\
10 \rightarrow(1,7), & l=5=1+4 & \text { [Fig. 9(j), P mode] }, \\
7 \rightarrow(2,2), & l=4=2+2 & \text { [Fig. 9(g), Q mode], } \\
7 \rightarrow(1,4), & l=4=1+3 & \text { [Fig. 9(g), Q mode] }, \\
4 \rightarrow(1,2), & l=3=1+2 & \text { [Fig. 9(d), T mode] },
\end{array}
$$

which may be understood as a kind of "weak" selection rule. However, since there is no conservation law for $l$, processes that increase or decrease $l$ also have nonzero amplitude; see, e.g., Fig. 9(b), E mode, $2 \rightarrow(3,4)$, for which $l$ increases.

This nonconservation is attributed to broken rotational symmetry. While an isolated skyrmion (with size much larger than the lattice spacing) is rotationally symmetric, which translates into conservation of azimuthal mode number $l$ similar to what was found for magnetic vortices [158], the skyrmions in a $\mathrm{SkX}$ experience a hexagonal deformation. With the necessary continuous symmetry broken, there is no conserved quantity.

\section{Decay channels analysis of $\mathrm{ABC}$ modes dependent on the magnetic field}

Here we study the magnetic-field dependence of the decay channels of the ABC modes. Results are presented in Fig. 10. As far as the mode labeling is concerned, recall that the flat polygon modes overtake the dispersive modes as the field increases, which is why the labels in Fig. 10 are different for different fields.

The field increases going from left to right. The instability of the $\mathrm{C}$ mode [Fig. 10(a)] towards the channel $\mathrm{C} \rightarrow\{\mathrm{A}, \mathrm{G}\}$ increases, while that towards the $\mathrm{C} \rightarrow\{\mathrm{E}, \mathrm{G}\}$ channel decreases. Other decay channels exhibit a weaker increase.

For the B mode [Fig. 10(b)] we find that channels towards lower-energy modes ( $\mathrm{G}, \mathrm{E}$, and $\mathrm{A})$ become less efficient at higher fields, resulting in an effective field-enhanced stability of the B mode, although decay channels towards higherenergy modes increase considerably.

Finally, we find that almost all decay channels of the A mode [Fig. 10(c)] get frozen out by the field. This complies with the finding that the A mode is the most stable mode among the ABC modes. Notice in particular that the decay channels towards lower-energy modes ( $\mathrm{G}$ and $\mathrm{E}$ modes) are always negligible, even at low fields. 

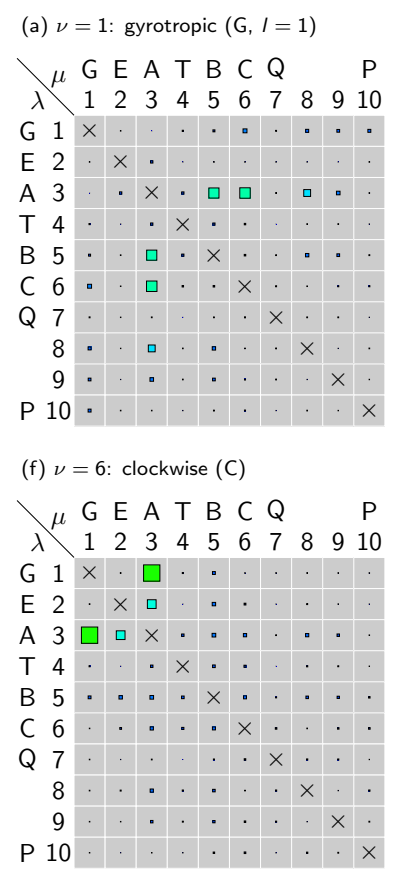

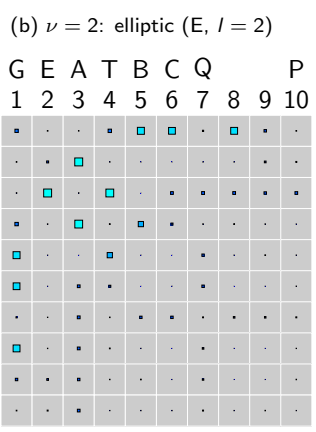

(g) $\nu=7$ : quadrupolar $(\mathrm{Q}, I=4)$

$\begin{array}{ccccccccccc}G & E & A & T & B & C & Q & & & P \\ 1 & 2 & 3 & 4 & 5 & 6 & 7 & 8 & 9 & 10\end{array}$

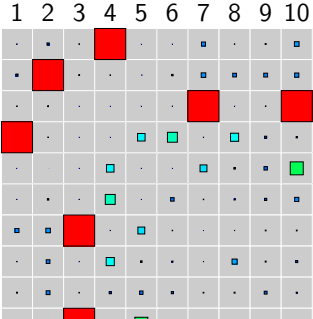

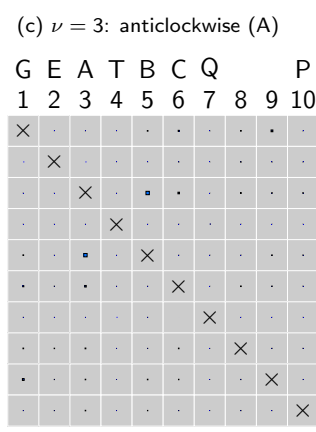

(h) $\nu=8$

G E A T B C Q P

$\begin{array}{llllllllll}1 & 2 & 3 & 4 & 5 & 6 & 7 & 8 & 9 & 10\end{array}$

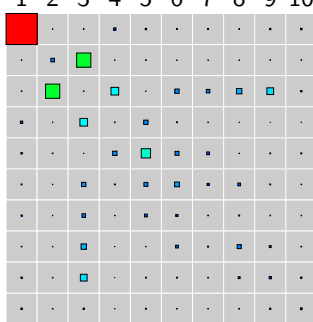

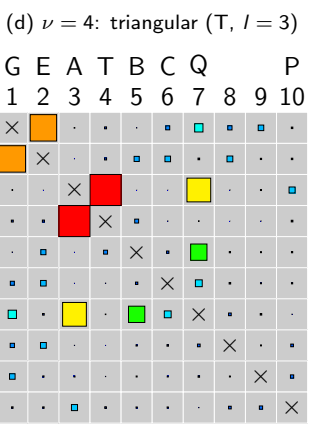

(i) $\nu=9$

G E A T B C Q P

$\begin{array}{llllllllll}1 & 2 & 3 & 4 & 5 & 6 & 7 & 8 & 9 & 10\end{array}$

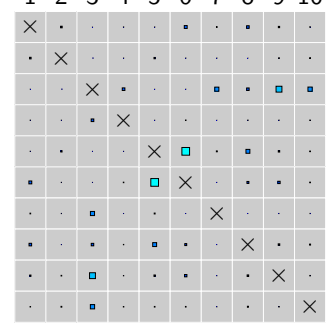

(e) $\nu=5$ : breathing $(\mathrm{B})$

G E A T B C Q

$\begin{array}{llllllllll}1 & 2 & 3 & 4 & 5 & 6 & 7 & 8 & 9 & 10\end{array}$

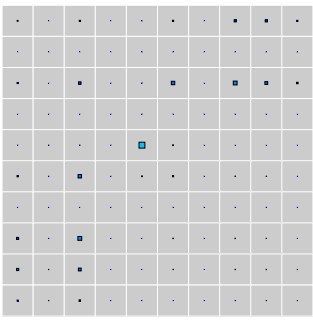

(j) $\nu=10$ : pentagonal $(\mathrm{P}, I=5)$

G E A T B C Q P

$\begin{array}{lllllllllll}1 & 2 & 3 & 4 & 5 & 6 & 7 & 8 & 9 & 10\end{array}$

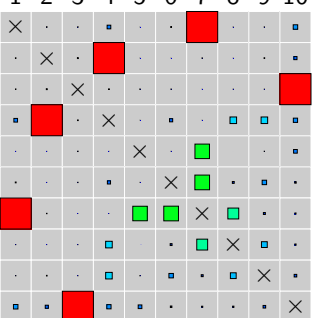

FIG. 9. Decay channel analysis of an $M=8 \mathrm{SkX}$ at $b / J S=0.5$ and $\boldsymbol{k}=\mathbf{0}$. (a)-(j) Decay channel efficiency $C^{\lambda \mu \leftarrow v}$ for $\lambda, \mu, v=1, \ldots, 10$ : (a) $v=1$, gyrotropic mode, $l=1$; (b) $v=2$, elliptic mode, $l=2$; (c) $v=3$, anticlockwise mode; (d) $v=4$, triangular mode, $l=3$; (e) $v=5$, breathing mode; (f) $v=6$, clockwise mode; (g) $v=7$, quadrupolar mode, $l=4$; (h) $v=8$; (i) $v=9$; and (j) $v=10$, pentagonal mode, $l=5$. Small blue/large red squares indicate small/large $C^{\lambda \mu \leftarrow \nu}$. The symbol $\times$ indicates exactly zero $C^{\lambda \mu \leftarrow \nu}$. Notice the decay product symmetry $\lambda \leftrightarrow \mu$.

\section{Effects of finite temperature on magnon damping}

At finite $T$, one obtains not only additional thermal weighting of the decay processes [first line in Eq. (A49)], but also additional collision processes (second line). Here we evaluate the temperature dependence of the damping of the ABC modes $(v=3,5,6$ at $b / J S=0.485)$. We decompose the total damping as

$$
\Gamma_{\mathbf{0}, v}^{\text {total }}(T)=\Gamma_{\mathbf{0}, v}^{\text {spon }}+\Gamma_{\mathbf{0}, v}^{\text {decay }}(T)+\Gamma_{\mathbf{0}, v}^{\text {coll }}(T),
$$

where the spontaneous part $\Gamma_{\mathbf{0}, v}^{\text {spon }}$ is what we have discussed so far,

$$
\begin{aligned}
\Gamma_{\mathbf{0}, v}^{\text {decay }}(T)= & \frac{\pi}{2 N_{\mathrm{u}}} \sum_{\boldsymbol{q}} \sum_{\lambda, \mu}\left|\mathcal{V}_{\boldsymbol{q},-\boldsymbol{q} \leftarrow \mathbf{0}}^{\lambda \mu \leftarrow v}\right|^{2} \delta\left(\varepsilon_{\mathbf{0}, v}-\varepsilon_{\boldsymbol{q}, \lambda}-\varepsilon_{-\boldsymbol{q}, \mu}\right) \\
& \times\left[\rho\left(\varepsilon_{\boldsymbol{q}, \lambda}, T\right)+\rho\left(\varepsilon_{-\boldsymbol{q}, \mu}, T\right)\right]
\end{aligned}
$$

is damping due to thermally activated decay processes, and

$$
\begin{aligned}
\Gamma_{\mathbf{0}, v}^{\mathrm{coll}}(T)= & \frac{\pi}{N_{\mathrm{u}}} \sum_{\boldsymbol{q}} \sum_{\lambda, \mu}\left|\mathcal{V}_{\mathbf{0}, \boldsymbol{q} \leftarrow \boldsymbol{q}}^{v \mu \leftarrow \lambda}\right|^{2} \delta\left(\varepsilon_{\mathbf{0}, \nu}+\varepsilon_{\boldsymbol{q}, \mu}-\varepsilon_{\boldsymbol{q}, \lambda}\right) \\
& \times\left[\rho\left(\varepsilon_{\boldsymbol{q}, \mu}, T\right)-\rho\left(\varepsilon_{\boldsymbol{q}, \lambda}, T\right)\right]
\end{aligned}
$$

is damping due to thermally activated collisions.

We choose a field $b / J S=0.485$, for which the spontaneous damping of the $\mathrm{C}$ mode is maximal [cf. Fig. 3(c)]. Results for the total damping dependent on temperature are shown in Fig. 11(a). As expected, finite temperatures lead to an increase of magnon damping.

As shown in Fig. 11(b), the A mode (blue line) exhibits the largest relative increase in damping due to thermally activated decays, $\Gamma_{\mathbf{0}, v}^{\text {decay }}(T)$. This can be explained by the factor $\left[\rho\left(\varepsilon_{\boldsymbol{q}, \lambda}, T\right)+\rho\left(\varepsilon_{-\boldsymbol{q}, \mu}, T\right)\right]$ in Eq. (B2). If one of the two decay products happens to be the $\mathrm{G}$ mode, which is lowest in energy, the thermal Bose weight is large, especially for small $q$. However, if one of the decay products has low energy and low momentum, the energy and momentum of the other decay product have to be close to those of the decaying magnon, that is, within the same mode (but necessarily at lower energy). Such a decay process is sketched in Fig. 11(d). Reviewing the energy dispersion of the $\mathrm{ABC}$ modes, one finds that only that of the A mode is concave at the Brillouin zone center. Hence, the aforementioned type of thermally enhanced decay process can only happen for the A mode.

The scenario for damping due to collisions $\Gamma_{\mathbf{0}, v}^{\text {coll }}(T)$, shown in Fig. 11(c), is opposite; the A mode exhibits the lowest relative increase. The factor $\left[\rho\left(\varepsilon_{\boldsymbol{q}, \mu}, T\right)-\rho\left(\varepsilon_{\boldsymbol{q}, \lambda}, T\right)\right]$ in Eq. (B3) tells us that a low-energy magnon at $\varepsilon_{\boldsymbol{q}, \mu}$, which collides with the magnon at $\varepsilon_{\mathbf{0}, v}$ to form a $\varepsilon_{\boldsymbol{q}, \lambda}$ magnon, dominates the thermal weight. If this low-energy magnon is in the $G$ mode, its contribution in energy and momentum to the final magnon is small. Thus, the final magnon must have energy and momentum close to the $\varepsilon_{\mathbf{0}, v}$ magnon (but necessarily higher energy). Such states are only available for the convex $\mathrm{B}$ and $\mathrm{C}$ modes, as indicated in Fig. 11(e), but absent for the concave A mode.

Combined, thermal damping and collision processes lead to the rather similar temperature trend of the ABC modes' damping shown in Fig. 11(a). The takeaway message from this analysis is that the thermally enhanced damping of the $\mathrm{C}$ mode at $k_{\mathrm{B}} T / J S=0.2$ is still a factor of 2 smaller than its spontaneous damping at $T=0$ [cf. green line in Fig. 11(a)]. We conclude that the quantum damping is also observable for 


\begin{tabular}{llll} 
& & Magnet \\
\hline$b=0.2 /(J S)$ & $b=0.4 /(J S)$ & $b=0.6 /(J S)$
\end{tabular}

(a) clockwise mode (C)
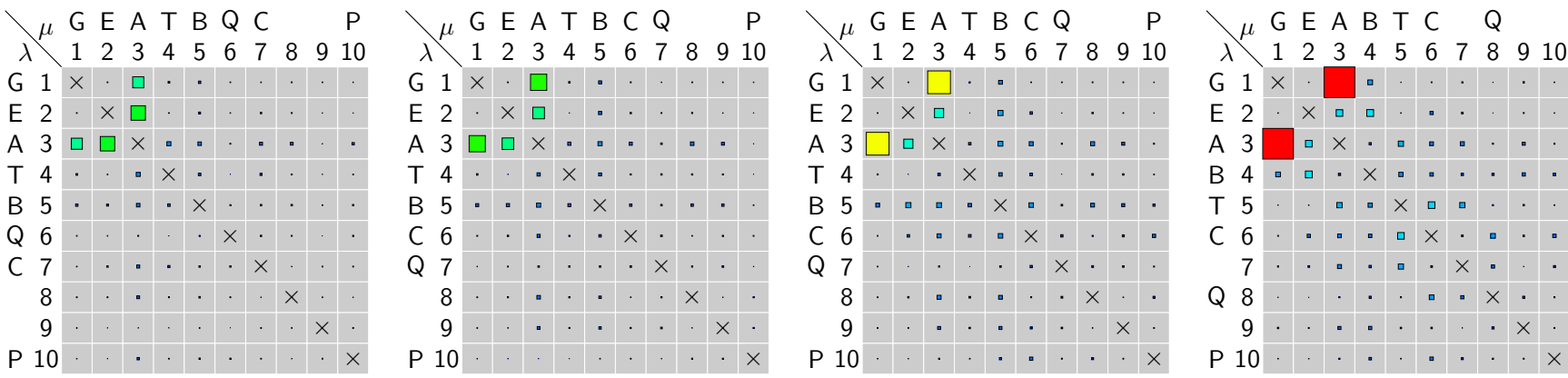

(b) breathing mode $(B)$
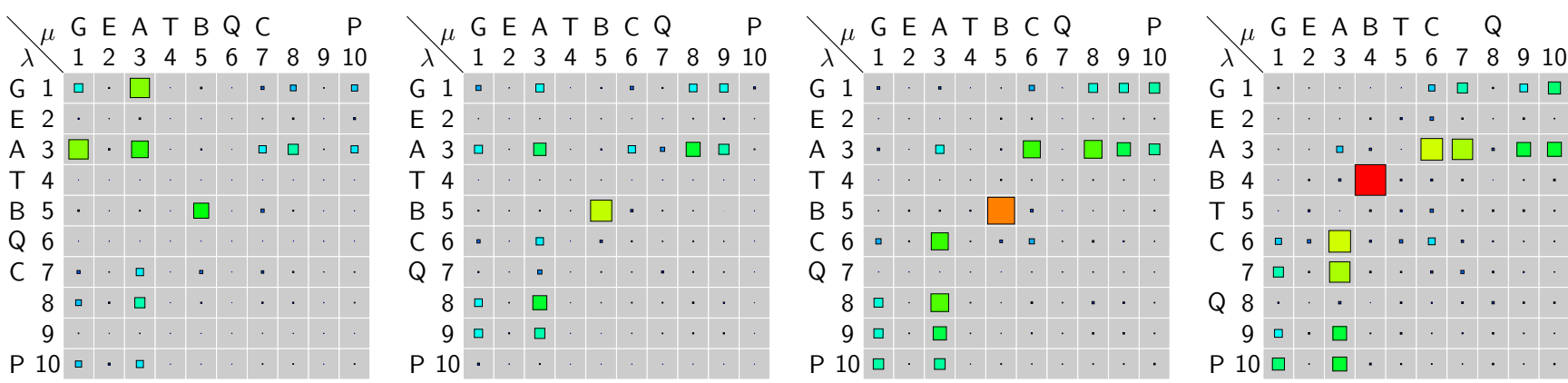

(c) anticlockwise mode $(A)$
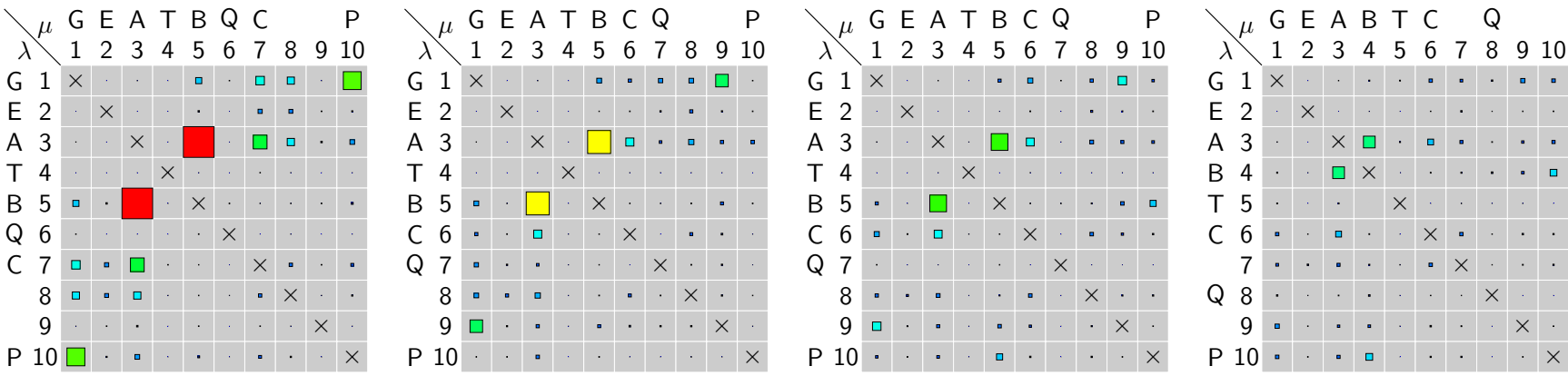

FIG. 10. Decay channel efficiencies $C^{\lambda \mu \leftarrow v}$ for the (a) C mode, (b) B mode, and (c) A mode dependent on magnetic field $b$. For each of the three modes, we used relative color scales, normalized to the largest $C^{\lambda \mu \leftarrow v}$ (large red squares).

finite-temperature SkXs, whose stability relies on moderate thermal fluctuations.

\section{Consistency of spin-wave theory}

The Mermin-Wagner theorem states that two-dimensional magnets with spin space isotropy exhibit no long-range order at nonzero temperatures [159]. Within spin-wave theory, as pioneered by Bloch [160], this is signaled by a diverging magnetization $M(T)$ for $T>0$, indicating the failure of the initial assumption of an ordered ground state.

For a chiral magnet, spin space isotropy is broken due to DM interaction and the Mermin-Wagner theorem does not apply. Nonetheless, we need to check for consistency to validate our spin-wave theory approach. We do so by noting that up to harmonic order the magnetization assumes the form

$$
M(T) \approx M_{0}+\delta M_{0}+M_{2}(T) .
$$

Here $M_{0}=-V^{-1} \partial E_{0} / \partial b$ is the classical ground-state magnetization obtained from the classical ground-state energy $E_{0}$ (or $\hat{H}_{0}$ ) and $V$ is the sample's volume. The lowest-order quantum correction to the magnetization $\delta M_{0}=-V^{-1} \partial \delta E_{0} / \partial b$ is associated with the harmonic zero-point energy $\delta E_{0}=$ $\sum_{v, k}\left[\varepsilon_{k, v}-\left(\mathbb{M}_{k}\right)_{v v}\right] / 2$, with $\mathbb{M}_{k}$ given in Eq. (A24a). Finally, $M_{2}(T)=-V^{-1} \sum_{v, \boldsymbol{k}} \rho\left(\varepsilon_{\boldsymbol{k}, v}, T\right) \partial \varepsilon_{\boldsymbol{k}, v} / \partial b$ accounts for the thermal occupation of magnons. Consistency demands that neither $\delta M_{0}$ nor $M_{2}(T)$ diverges, i.e., neither quantum nor thermal fluctuations destroy magnetic order.

Let us quickly review the failure of spin-wave theory for a two-dimensional ferromagnet with spin space isotropy [160]. 

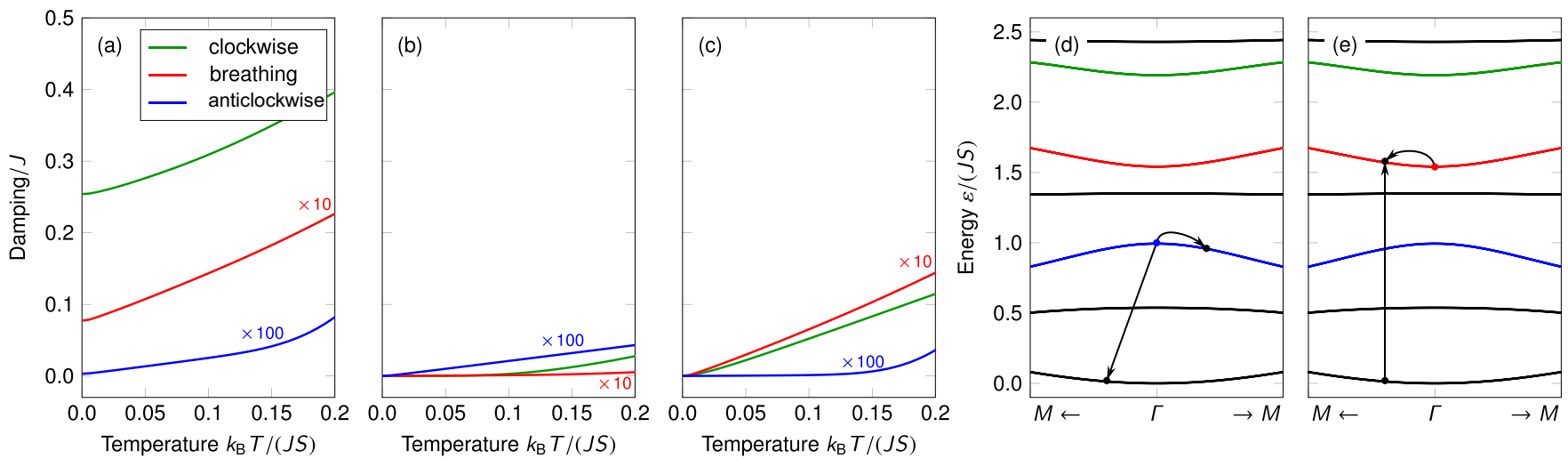

FIG. 11. Damping of ABC modes of an $M=8 \mathrm{SkX}$ at $b / J S=0.485$ dependent on temperature $T$. (a) Total damping $\Gamma_{\mathbf{0}}^{\text {total }}(T)=\Gamma_{\mathbf{0}}^{\text {spon }}+$ $\Gamma_{\mathbf{0}}^{\text {decay }}(T)+\Gamma_{\mathbf{0}}^{\text {coll }}(T)$, (b) damping $\Gamma_{\mathbf{0}}^{\text {decay }}(T)$ due to thermally activated decays, and (c) damping $\Gamma_{\mathbf{0}}^{\text {coll }}(T)$ due to thermally activated collisions. Also shown is the magnon band structure in the vicinity of the Brillouin zone center with (d) the exemplary decay process and (e) the exemplary collision process.

Since there are no quantum fluctuations $\left(\delta M_{0}=0\right)$, only

$$
M_{2}(T) \propto \sum_{n} \int_{\mathrm{BZ}} \frac{1}{e^{\varepsilon_{k, v} /\left(k_{\mathrm{B}} T\right)}-1} d^{2} k
$$

remains to be investigated. Integration is over the first Brillouin zone. (We used that $-\partial \varepsilon_{k, v} / \partial b$ is constant because all magnons carry the same nonzero magnetic moment.) The lowest-energy magnon with energy $\varepsilon_{\boldsymbol{k}, 1} \propto k^{2}$ is a Goldstone mode. Thus, for $T>0$, there is a region $k=|\boldsymbol{k}|<\Lambda$ for which $\varepsilon_{k, 1} \ll k_{\mathrm{B}} T$ ( $\Lambda>0$ being an upper cutoff). Consequently, after expanding the exponential in Eq. (B5), we find

$$
M_{2}(T) \propto T \int_{0}^{\Lambda} \frac{k}{\varepsilon_{k, 1}} d k \propto T \int_{0}^{\Lambda} \frac{1}{k} d k
$$

and encounter the divergence of thermal fluctuations.

For a SkX in the continuous limit, there is also a Goldstone mode with $\varepsilon_{k} \propto k^{2}$. It is associated with the spontaneously broken translational invariance. Hence, it costs no energy to rigidly translate the $\mathrm{SkX}$ in space. In our discrete simulations, translational invariance is already broken on the Hamiltonian level and the Goldstone mode becomes the gyrotropic mode with a tiny spin-wave gap. Nonetheless, we can extract its magnetic moment $-\partial \varepsilon_{\boldsymbol{k}, 1} / \partial b$, as depicted in Fig. 12(a). For

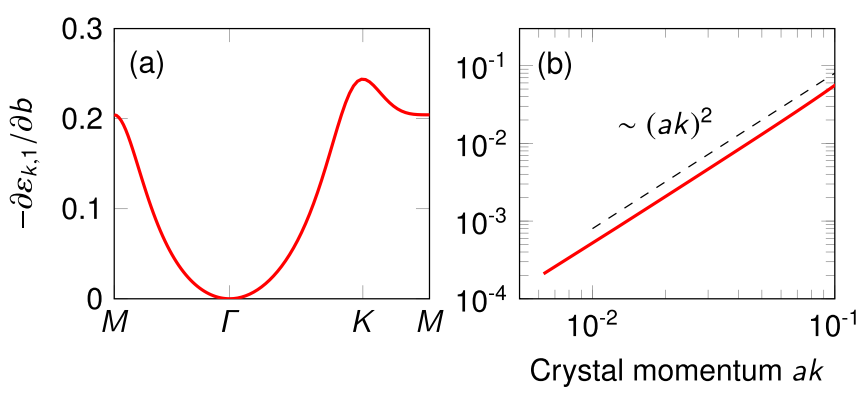

FIG. 12. Magnetic moment of a SkX's translational Goldstone mode (a) along high-symmetry paths and (b) dependent on the distance from the Brillouin zone center. low momenta, we find that is goes as $k^{2}$ [Fig. 12(b)]. Consequently, the divergence of the magnetization is lifted:

$$
M_{2}(T) \propto T \int_{0}^{\Lambda} \frac{1}{k} \frac{\partial \varepsilon_{k}}{\partial b} d k \propto T \int_{0}^{\Lambda} k d k<\infty .
$$

Since typical chiral magnets have small $D / J$ ratios, the crucial ingredient to break spin space isotropy is also small. This installs a threshold temperature above which spin-wave theory breaks down. The first almost flat magnon mode, which may exhibit a divergent thermal contribution to the magnetization, approximately appears at an energy $\varepsilon_{0}=J S(D / J)^{2}$. Due to the almost constant magnetic moment of flat modes, its thermal contribution to $M(T)$ reads

$$
M_{2}(T) \propto \rho\left(\varepsilon_{0}, T\right) \int_{\mathrm{BZ}} d^{2} k .
$$

In the limit of $D / J \ll 1$ and $\varepsilon_{0} \ll k_{\mathrm{B}} T$, we have $\rho\left(\varepsilon_{0}, T\right) \propto$ $\frac{k_{\mathrm{B}} T}{J S(D / J)^{2}}$ and the Brillouin zone area $\int_{\mathrm{BZ}} d^{2} k \propto(D / J)^{2}$; hence

$$
M_{2}(T) \propto \frac{k_{\mathrm{B}} T}{J S} .
$$

Notice that the $D / J$ ratio cancels out. Thus, spin-wave theory for a two-dimensional $\mathrm{SkX}$ is consistent if

$$
k_{\mathrm{B}} T \ll J S \text {. }
$$

Since $k_{\mathrm{B}} T_{\mathrm{c}} \sim J S$ is approximately the ordering temperature of the $\mathrm{SkX}$ [116], Eq. (B10) tells us that validity of (harmonic) spin-wave theory is restricted to temperatures well below the ordering temperature, which is the usual realm of applicability.

At $T=0$, we are still concerned with quantum fluctuations

$$
\delta M_{0}=-\frac{1}{2 V} \sum_{\nu, \boldsymbol{k}}\left(\frac{\partial \varepsilon_{\boldsymbol{k}, v}}{\partial b}-\frac{\partial\left(\mathbb{M}_{\boldsymbol{k}}\right)_{\nu v}}{\partial b}\right)
$$

due to the noncollinearity of the SkX. Note that there are no divergent contributions in Eq. (B11). This is verified by direct numerical evaluation. In Fig. 13 we show the ratio $\delta M_{0} / S M_{0}$, encoding the relative importance of quantum corrections with respect to the classical ground-state magnetization $M_{0}$, dependent on the skyrmion unit cell size. Even for tiny skyrmion 
Linear size of skyrmion unit cell $M$

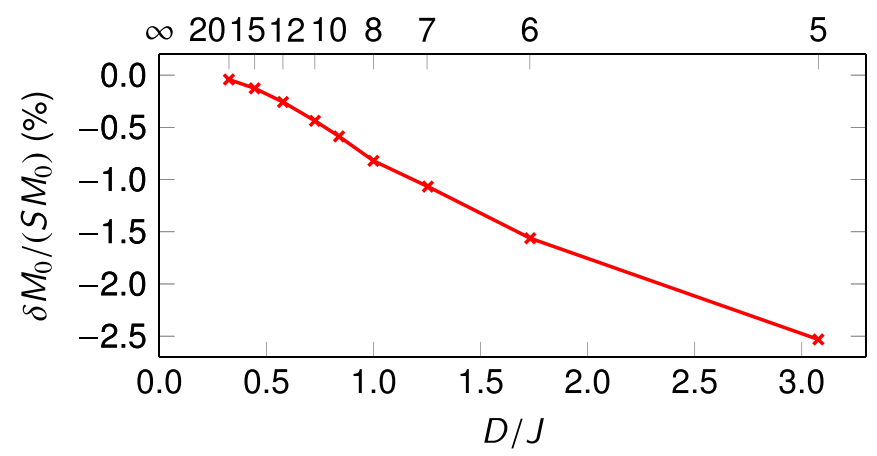

FIG. 13. Ratio of quantum corrections to the magnetization $\delta M_{0}$ and the classical ground-state magnetization $M_{0}$ dependent on $D / J$ (or linear skyrmion size $M$ ). We set $b / J S=0.5(D / J)^{2}$.

unit cells with a linear size of $M=5$ (25 spins per unit cell), the quantum mechanical magnetization corrections are smaller than $3 \%$ (for $S=1$ ). We conclude that quantum fluctuations are negligible.

\section{Dependence of quantum damping on skyrmion size}

In Fig. 14 we plot the total three-magnon vertex

$$
V_{\boldsymbol{k}, \boldsymbol{q} \leftarrow \boldsymbol{k}+\boldsymbol{q}}^{\mathrm{total}} \equiv \sum_{\lambda, \mu, \nu} V_{\boldsymbol{k}, \boldsymbol{q} \leftarrow \boldsymbol{k}+\boldsymbol{q}}^{\lambda \mu \leftarrow v}=-\sum_{\lambda, \mu, \nu}\left(\delta_{\mu \nu} \mathcal{I}_{\boldsymbol{k}, \lambda \mu}^{+z}+\delta_{\lambda \nu} \mathcal{I}_{\boldsymbol{q}, \mu \lambda}^{+z}\right)
$$

per magnetic unit cell at zero momenta $(\boldsymbol{k}=\boldsymbol{q}=\mathbf{0})$, i.e.,

$$
V_{\mathbf{0}, \mathbf{0} \leftarrow \mathbf{0}}^{\mathrm{total}}=-2 \sum_{\mu, v} \mathcal{I}_{\mathbf{0}, \mu \nu}^{+z},
$$

dependent on the $D / J$ ratio (skyrmion size). As $D / J \rightarrow 0$, a single skyrmion becomes infinitely large, but the total threemagnon vertex $V_{\mathbf{0}, \mathbf{0} \leftarrow \mathbf{0}}^{\text {total }}$ converges to a nonzero constant. Thus,

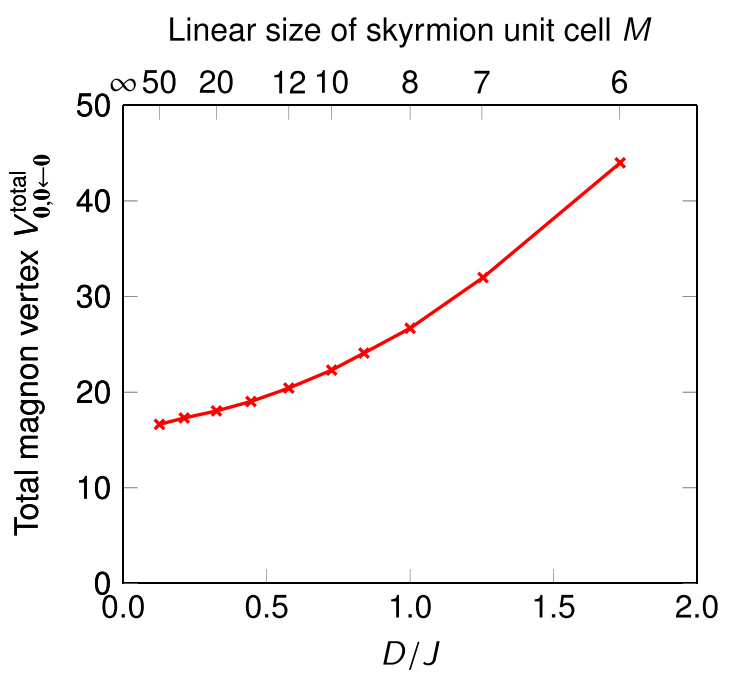

FIG. 14. Integrated three-magnon vertex dependent on $D / J$ (bottom abscissa) and linear skyrmion size $M$ (top abscissa). We set $b / J S=0.5(D / J)^{2}$. the averaged three-magnon vertex

$$
\overline{V_{\mathbf{0 , 0} \mathbf{0} \leftarrow \mathbf{0}}^{\lambda \mu \leftarrow v}} \equiv \frac{1}{6} \frac{V_{\mathbf{0 , 0} \mathbf{0}}^{\text {total }}}{N} \approx \frac{1}{6} \frac{V_{\mathbf{0 , 0} \mathbf{0} \leftarrow \mathbf{0}}^{\text {total }}}{\left[2 \pi / \arctan \left(\frac{D}{J}\right)\right]^{2}} \propto J\left(\frac{D}{J}\right)^{2}
$$

scales with $J(D / J)^{2}$ if $D / J \ll 1$. The same finding applies to finite momenta, which merely introduce phase factors. (The factor $\frac{1}{6}$ is due to the six nearest neighbors on the triangular lattice.)

In the limit of large skyrmions, the degree of noncollinearity is tiny, which means for the spin-wave kernel $\mathbb{H}_{k}$ in Eq. (A23) that the anomalous coupling matrix $\mathbb{N}_{k} \propto D / J$ is tiny. Consequently, the $\mathbb{Y}_{\boldsymbol{k}} \propto D / J$ submatrix of $\mathbb{P}_{\boldsymbol{k}}$ in Eq. (A31) is tiny too. As for the decay vertex in Eq. (A44), we conclude that on average

$$
\overline{\mathcal{V}_{\boldsymbol{k}, \boldsymbol{q} \leftarrow \boldsymbol{k}+\boldsymbol{q}}^{\lambda \mu \leftarrow \nu}} \approx \sum_{\alpha, \beta, \gamma} \overline{V_{\boldsymbol{k}, \boldsymbol{q} \leftarrow \boldsymbol{k}+\boldsymbol{q}}^{\alpha \beta \leftarrow \gamma}}\left(\mathbb{X}_{\boldsymbol{k}}^{*}\right)_{\lambda \alpha}\left(\mathbb{X}_{\boldsymbol{q}}^{*}\right)_{\mu \beta}\left(\mathbb{X}_{\boldsymbol{p}}\right)_{\nu \gamma} \propto J\left(\frac{D}{J}\right)^{2}
$$

is the dominating contribution because $\mathbb{X}_{k} \propto(D / J)^{0}$. Introducing the mean decay amplitude as

$$
\left|\overline{\mathcal{V}_{\boldsymbol{k}}^{v}}\right|^{2} \equiv \frac{1}{N_{\mathrm{u}}} \frac{1}{N^{2}} \sum_{\boldsymbol{q}} \sum_{\lambda, \mu}\left|\overline{\mathcal{V}_{\boldsymbol{k}, \boldsymbol{q} \leftarrow \boldsymbol{k}+\boldsymbol{q}}^{\lambda \mu \leftarrow v}}\right|^{2} \propto J^{2}\left(\frac{D}{J}\right)^{4}
$$

(note that the sum over $\lambda$ and $\mu$ and the $1 / N^{2}$ factor have inverse scaling with $D / J$ such that their influence cancels out), we find for the spontaneous damping that

$$
\Gamma_{\boldsymbol{k}, v}^{\text {spon }} \approx \frac{\pi}{2}\left|\overline{\mathcal{V}_{\boldsymbol{k}}^{v}}\right|^{2} \mathcal{D}_{\boldsymbol{k}}^{2 \mathrm{~m}}\left(\varepsilon_{v, \boldsymbol{k}}\right) \propto J\left(\frac{D}{J}\right)^{4} \text { as } \frac{D}{J} \rightarrow 0
$$

because $\mathcal{D}_{k}^{2 \mathrm{~m}} \propto 1 / J$. We use Eq. (B17) to estimate the damping of the ABC modes $\Gamma_{X}^{\text {spon }}$, with $X=\mathrm{A}, \mathrm{B}, \mathrm{C}$. As shown in Fig. 3(c), $\Gamma_{X}^{\text {spon }} \approx F_{X} J$, with $F_{\mathrm{A}}=10^{-3}, F_{\mathrm{B}}=10^{-2}$, and $F_{\mathrm{C}}=$ $10^{-1}$ (for $\left.D / J=1\right)$. Hence, we expect $\Gamma_{X}^{\text {spon }} \approx F_{X} J(D / J)^{4}$.

\section{Influence of dimensionality on magnon damping}

As mentioned in the main text, the zero-temperature damping is directly related to the two-magnon density of states, which derives from the $\delta$ function in Eq. (A49) (first sum). In two dimensions, the onset of the two-magnon DOS is a Heaviside step function. Thus, as a single-particle band crosses the onset of the two-magnon DOS, its damping also exhibits a step, which renders the magnon damping and linewidth broadening discontinuous. In contrast, in three dimensions, the onset is given by a square root (see also Refs. [95,97]). Hence, if instead of a two-dimensional SkX we studied a three-dimensional SkX, e.g., built from stacking SkX layers, we would expect a renormalization of the step. However, this renormalization is not as drastic as that associated with going from one to two dimensions, for which the Van Hove singularity of the two-magnon DOS changes from an inverse square root to the step function [97]. Thus, in general, the overall magnitude of the spontaneous magnon damping is not expected to be considerably smaller in three than in two dimensions. Hence, details become important, above all the magnetic texture along the third 
dimension. For example, skyrmion tubes, which are stacks of two-dimensional skyrmion crystals, are expected to exhibit spontaneous decay different from that of Bloch point crystals, which are genuinely three-dimensional noncollinear textures. For a noncollinear order along the third direction, larger contributions to the three-magnon vertices are expected than for a collinear order.

There is another important aspect of three-dimensional SkXs, associated with the magnon bands acquiring dispersion along the third direction [161]. For quasi-two-dimensional layered structures with weak interlayer coupling much smaller than the size of typical magnon band gaps in two dimensions, the dispersion along the additional dimension is negligible. However, for interlayer coupling as large as (or larger than) magnon band gaps, the flat bands in two dimensions can no longer be considered flat and their influence on magnon damping becomes less abrupt.

For a real sample of finite thickness $L$, we expect a transition between two-dimensional and three-dimensional behavior with increasing thickness. The finite thickness acts as a quantum well, introducing several magnon energy levels

$$
E_{n}^{j}=\varepsilon_{n}^{2 \mathrm{D}}+\frac{j^{2} \pi^{2} \hbar^{2}}{2 m^{*} L^{2}},
$$

with quantum number $j$ for each magnon band $n$ with energy $\varepsilon_{n}^{2 \mathrm{D}}$ in two dimensions. We assumed a parabolic dispersion along the third direction, ignoring subleading nonreciprocal shifts due to DMI. The respective effective magnon mass $m^{*}=\hbar^{2} / 2 S J_{\perp} d^{2}$ includes the exchange interaction $J_{\perp}$ along the third direction and the layer spacing $d$. The $E_{n}^{j}$ 's become dense as $L \rightarrow \infty$ and periodic boundary conditions may be applied. A critical thickness $L^{\prime}$ may be defined by the condition that the broadening of the discrete magnon quantum well levels, due to either spontaneous decay or Gilbert damping, equals their energetic spacing $\Delta E \approx S J_{\perp}(\pi d / L)^{2}$, which we approximate by setting $j=1$. For the classical Gilbert damping, the magnon damping reads $\Gamma_{n, j}^{\alpha} \approx \alpha E_{n}^{j} \approx \alpha \varepsilon_{n}^{2 \mathrm{D}}$. The associated critical thickness is thus

$$
L_{\mathrm{class}}^{\prime} \approx \pi \sqrt{\frac{S J_{\perp}}{\alpha \varepsilon_{n}^{2 \mathrm{D}}}} d .
$$

Taking the $\mathrm{C}$ mode as reference, we have $\varepsilon_{n}^{2 \mathrm{D}} \approx J S(D / J)^{2}$. With $J_{\perp}=J$, as expected for a cubic crystal, it follows that

$$
L_{\text {class }}^{\prime} \approx \frac{\pi}{\sqrt{\alpha}} \frac{J}{D} d,
$$

which amounts to $L_{\text {class }}^{\prime} \sim 5000 d$ for parameters of $\mathrm{Cu}_{2} \mathrm{OSeO}_{3} \quad\left(\alpha=10^{-4}\right.$ and $D / J \approx 0.06$, taken from Refs. [138] and [8], respectively). In contrast, for the spontaneous quantum damping, we set $\Gamma_{\mathrm{C}}^{\text {spon }} \approx 0.1 J(D / J)^{4}$ (see Appendix B 6) and find

$$
L_{\mathrm{spon}}^{\prime} \approx \pi \sqrt{\frac{10 S J_{\perp}}{J}}\left(\frac{J}{D}\right)^{2} d .
$$

With $S=1$ and $J_{\perp}=J$, we obtain $L_{\text {spon }}^{\prime} \sim 2700 d$ for $\mathrm{Cu}_{2} \mathrm{OSeO}_{3}$. In terms of the relative importance of quantum to classical damping $R_{\mathrm{C}}$, as introduced in Sec. IV, the relation

$$
L_{\text {class }}^{\prime}=\sqrt{R_{\mathrm{C}}} L_{\mathrm{spon}}^{\prime}
$$

is extracted. Obviously, if the quantum damping is stronger than the classical damping, it sets the critical thickness for the crossover between two-dimensional and three-dimensional behavior.

We conclude that the results on spontaneous decay we have obtained in the strictly two-dimensional limit apply to thin films with a thickness $L \ll \min \left(L_{\text {class }}^{\prime}, L_{\text {spon }}^{\prime}\right)$.
[1] A. N. Bogdanov and D. Yablonskii, Zh. Eksp. Teor. Fiz. 95, 178 (1989).

[2] A. N. Bogdanov and U. K. Rößler, Phys. Rev. Lett. 87, 037203 (2001).

[3] U. K. Rößler, A. N. Bogdanov, and C. Pfleiderer, Nature (London) 442, 797 (2006).

[4] S. Mühlbauer, B. Binz, F. Jonietz, C. Pfleiderer, A. Rosch, A. Neubauer, R. Georgii, and P. Böni, Science 323, 915 (2009).

[5] X. Z. Yu, Y. Onose, N. Kanazawa, J. H. Park, J. H. Han, Y. Matsui, N. Nagaosa, and Y. Tokura, Nature (London) 465, 901 (2010).

[6] W. Münzer, A. Neubauer, T. Adams, S. Mühlbauer, C. Franz, F. Jonietz, R. Georgii, P. Böni, B. Pedersen, M. Schmidt, A. Rosch, and C. Pfleiderer, Phys. Rev. B 81, 041203(R) (2010).

[7] X. Z. Yu, N. Kanazawa, Y. Onose, K. Kimoto, W. Z. Zhang, S. Ishiwata, Y. Matsui, and Y. Tokura, Nat. Mater. 10, 106 (2011).

[8] S. Seki, X. Z. Yu, S. Ishiwata, and Y. Tokura, Science 336, 198 (2012).

[9] I. Kézsmárki, S. Bordács, P. Milde, E. Neuber, L. M. Eng, J. S. White, H. M. Rønnow, C. D. Dewhurst, M. Mochizuki,
K. Yanai, H. Nakamura, D. Ehlers, V. Tsurkan, and A. Loidl, Nat. Mater. 14, 1116 (2015).

[10] G. Finocchio, F. Büttner, R. Tomasello, M. Carpentieri, and M. Kläui, J. Phys. D 49, 423001 (2016).

[11] S. Seki and M. Mochizuki, Skyrmions in Magnetic Materials (Springer International, Cham, 2016).

[12] A. Soumyanarayanan, M. Raju, A. L. Gonzalez Oyarce, A. K. C. Tan, M.-Y. Im, A. P. Petrović, P. Ho, K. H. Khoo, M. Tran, C. K. Gan, F. Ernult, and C. Panagopoulos, Nat. Mater. 16, 898 (2017).

[13] A. K. Nayak, V. Kumar, T. Ma, P. Werner, E. Pippel, R. Sahoo, F. Damay, U. K. Rößler, C. Felser, and S. S. P. Parkin, Nature (London) 548, 561 (2017).

[14] T. Kurumaji, T. Nakajima, V. Ukleev, A. Feoktystov, T.-h. Arima, K. Kakurai, and Y. Tokura, Phys. Rev. Lett. 119, 237201 (2017).

[15] A. Fert, N. Reyren, and V. Cros, Nat. Rev. Mater. 2, 17031 (2017).

[16] W. Jiang, G. Chen, K. Liu, J. Zang, S. G. te Velthuis, and A. Hoffmann, Phys. Rep. 704, 1 (2017). 
[17] Y. Zhou, Natl. Sci. Rev. 6, 210 (2018).

[18] K. Everschor-Sitte, J. Masell, R. M. Reeve, and M. Kläui, J. Appl. Phys. 124, 240901 (2018).

[19] B. Binz and A. Vishwanath, Physica B 403, 1336 (2008).

[20] M. Lee, W. Kang, Y. Onose, Y. Tokura, and N. P. Ong, Phys. Rev. Lett. 102, 186601 (2009).

[21] A. Neubauer, C. Pfleiderer, B. Binz, A. Rosch, R. Ritz, P. G. Niklowitz, and P. Böni, Phys. Rev. Lett. 102, 186602 (2009).

[22] T. Schulz, R. Ritz, A. Bauer, M. Halder, M. Wagner, C. Franz, C. Pfleiderer, K. Everschor, M. Garst, and A. Rosch, Nat. Phys. 8, 301 (2012).

[23] M. Mochizuki, Phys. Rev. Lett. 108, 017601 (2012).

[24] Y. Li, N. Kanazawa, X. Z. Yu, A. Tsukazaki, M. Kawasaki, M. Ichikawa, X. F. Jin, F. Kagawa, and Y. Tokura, Phys. Rev. Lett. 110, 117202 (2013).

[25] K. A. van Hoogdalem, Y. Tserkovnyak, and D. Loss, Phys. Rev. B 87, 024402 (2013).

[26] M. Mochizuki, X. Z. Yu, S. Seki, N. Kanazawa, W. Koshibae, J. Zang, M. Mostovoy, Y. Tokura, and N. Nagaosa, Nat. Mater. 13, 241 (2014).

[27] A. Mook, B. Göbel, J. Henk, and I. Mertig, Phys. Rev. B 95, 020401(R) (2017).

[28] D.-S. Han, K. Lee, J.-P. Hanke, Y. Mokrousov, K.-W. Kim, W. Yoo, Y. L. W. van Hees, T.-W. Kim, R. Lavrijsen, C.-Y. You, H. J. M. Swagten, M.-H. Jung, and M. Kläui, Nat. Mater. 18, 703 (2019).

[29] J. Iwasaki, M. Mochizuki, and N. Nagaosa, Nat. Nanotechnol. 8, 742 (2013).

[30] R. Tomasello, E. Martinez, R. Zivieri, L. Torres, M. Carpentieri, and G. Finocchio, Sci. Rep. 4, 6784 (2014).

[31] B. S. Kim, J. Phys.: Condens. Matter 31, 383001 (2019).

[32] X. Zhang, M. Ezawa, and Y. Zhou, Sci. Rep. 5, 9400 (2015).

[33] S. Luo, M. Song, X. Li, Y. Zhang, J. Hong, X. Yang, X. Zou, N. Xu, and L. You, Nano Lett. 18, 1180 (2018).

[34] W. Kang, X. Chen, D. Zhu, S. Li, Y. Huang, Y. Zhang, and W. Zhao, in Proceedings of the 2018 Design, Automation and Test in Europe Conference Exhibition (DATE) (IEEE, Piscataway, NJ, 2018), pp. 119-124.

[35] D. Pinna, G. Bourianoff, and K. Everschor-Sitte, arXiv:1811.12623.

[36] J. Zázvorka, F. Jakobs, D. Heinze, N. Keil, S. Kromin, S. Jaiswal, K. Litzius, G. Jakob, P. Virnau, D. Pinna, K. Everschor-Sitte, L. Rózsa, A. Donges, U. Nowak, and M. Kläui, Nat. Nanotechnol. 14, 658 (2019).

[37] M. G. Mankalale, Z. Zhao, J. Wang, and S. S. Sapatnekar, IEEE Trans. Electron Devices 66, 1990 (2019).

[38] J. Graf, H. Pfeifer, F. Marquardt, and S. Viola Kusminskiy, Phys. Rev. B 98, 241406(R) (2018).

[39] M. J. Martínez-Pérez and D. Zueco, New J. Phys. 21, 115002 (2019).

[40] S. V. Kusminskiy, Quantum Magnetism, Spin Waves, and Optical Cavities, SpringerBriefs in Physics (Springer International, Cham, 2019), pp. 71-87.

[41] C. Schütte and M. Garst, Phys. Rev. B 90, 094423 (2014).

[42] F. Ma, Y. Zhou, and W. S. Lew, IEEE Trans. Magn. 51, 1 (2015).

[43] F. Ma, Y. Zhou, H. B. Braun, and W. S. Lew, Nano Lett. 15, 4029 (2015).

[44] A. Roldán-Molina, A. S. Nunez, and J. Fernández-Rossier, New J. Phys. 18, 045015 (2016).
[45] P. M. Buhl, F. Freimuth, S. Blügel, and Y. Mokrousov, Phys. Status Solidi 11, 1700007 (2017).

[46] B. Göbel, A. Mook, J. Henk, and I. Mertig, Phys. Rev. B 96, 060406(R) (2017).

[47] X. Zhang, J. Müller, J. Xia, M. Garst, X. Liu, and Y. Zhou, New J. Phys. 19, 065001 (2017).

[48] M. Garst, J. Waizner, and D. Grundler, J. Phys. D 50, 293002 (2017).

[49] S. A. Díaz, J. Klinovaja, and D. Loss, Phys. Rev. Lett. 122, 187203 (2019).

[50] S.-Z. Lin, J.-X. Zhu, and A. Saxena, Phys. Rev. B 99, 140408(R) (2019).

[51] S. K. Kim, K. Nakata, D. Loss, and Y. Tserkovnyak, Phys. Rev. Lett. 122, 057204 (2019).

[52] S. A. Díaz, T. Hirosawa, J. Klinovaja, and D. Loss, Phys. Rev. Research 2, 013231 (2020).

[53] X. Xing, Y. Zhou, and H. B. Braun, Phys. Rev. Appl. 13, 034051 (2020).

[54] L. Zhang, J. Ren, J.-S. Wang, and B. Li, Phys. Rev. B 87, 144101 (2013).

[55] R. Shindou, R. Matsumoto, S. Murakami, and J.-i. Ohe, Phys. Rev. B 87, 174427 (2013).

[56] A. Mook, J. Henk, and I. Mertig, Phys. Rev. B 90, 024412 (2014).

[57] X. S. Wang, H. W. Zhang, and X. R. Wang, Phys. Rev. Appl. 9, 024029 (2018).

[58] S.-Z. Lin and L. N. Bulaevskii, Phys. Rev. B 88, 060404(R) (2013).

[59] A. Roldán-Molina, M. J. Santander, A. S. Nunez, and J. Fernández-Rossier, Phys. Rev. B 92, 245436 (2015).

[60] R. Takashima, H. Ishizuka, and L. Balents, Phys. Rev. B 94, 134415 (2016).

[61] D. N. Aristov and P. G. Matveeva, Phys. Rev. B 94, 214425 (2016).

[62] S. A. Diaz and D. P. Arovas, arXiv:1604.04010.

[63] C. Psaroudaki, S. Hoffman, J. Klinovaja, and D. Loss, Phys. Rev. X 7, 041045 (2017).

[64] B. Douçot, D. L. Kovrizhin, and R. Moessner, Ann. Phys. (NY) 399, 239 (2018).

[65] A. Derras-Chouk, E. M. Chudnovsky, and D. A. Garanin, Phys. Rev. B 98, 024423 (2018).

[66] C. Psaroudaki and D. Loss, Phys. Rev. Lett. 120, 237203 (2018)

[67] O. M. Sotnikov, V. V. Mazurenko, J. Colbois, F. Mila, M. I. Katsnelson, and E. A. Stepanov, arXiv:1811.10823.

[68] H. Ochoa and Y. Tserkovnyak, Int. J. Mod. Phys. B 33, 1930005 (2019).

[69] C. Psaroudaki, P. Aseev, and D. Loss, Phys. Rev. B 100, 134404 (2019).

[70] V. Lohani, C. Hickey, J. Masell, and A. Rosch, Phys. Rev. X 9, 041063 (2019).

[71] S. M. Vlasov, P. F. Bessarab, I. S. Lobanov, M. N. Potkina, V. M. Uzdin, and H. Jónsson, New J. Phys. 22, 083013 (2020).

[72] C. Psaroudaki and D. Loss, Phys. Rev. Lett. 124, 097202 (2020).

[73] L. P. Pitaevskii, Zh. Eksp. Teor. Fiz. 36, 1168 (1959) [Sov. Phys. JETP 9, 830 (1959)].

[74] A. Zawadowski, J. Ruvalds, and J. Solana, Phys. Rev. A 5, 399 (1972). 
[75] E. H. Graf, V. J. Minkiewicz, H. B. Møller, and L. Passell, Phys. Rev. A 10, 1748 (1974).

[76] A. J. Smith, R. A. Cowley, A. D. B. Woods, W. G. Stirling, and P. Martel, J. Phys. C 10, 543 (1977).

[77] R. Baumgartner, M. Engelhardt, and K. F. Renk, Phys. Rev. Lett. 47, 1403 (1981).

[78] Phonon Scattering in Condensed Matter, edited by W. Eisenmenger, K. Laßmann, and S. Döttinger (Springer, Berlin, 1984).

[79] H. R. Glyde, M. R. Gibbs, W. G. Stirling, and M. A. Adams, Europhys. Lett. 43, 422 (1998).

[80] B. Fåk and J. Bossy, J. Low Temp. Phys. 113, 531 (1998).

[81] M. E. Zhitomirsky and A. L. Chernyshev, Phys. Rev. Lett. 82, 4536 (1999).

[82] M. E. Zhitomirsky, Phys. Rev. B 73, 100404(R) (2006).

[83] M. B. Stone, I. A. Zaliznyak, T. Hong, C. L. Broholm, and D. H. Reich, Nature (London) 440, 187 (2006).

[84] A. L. Chernyshev and M. E. Zhitomirsky, Phys. Rev. Lett. 97, 207202 (2006)

[85] O. F. Syljuåsen, Phys. Rev. B 78, 180413(R) (2008).

[86] A. Kreisel, F. Sauli, N. Hasselmann, and P. Kopietz, Phys. Rev. B 78, 035127 (2008).

[87] A. Lüscher and A. M. Läuchli, Phys. Rev. B 79, 195102 (2009).

[88] A. L. Chernyshev and M. E. Zhitomirsky, Phys. Rev. B 79, 144416 (2009).

[89] M. Mourigal, M. E. Zhitomirsky, and A. L. Chernyshev, Phys. Rev. B 82, 144402 (2010).

[90] T. Masuda, S. Kitaoka, S. Takamizawa, N. Metoki, K. Kaneko, K. C. Rule, K. Kiefer, H. Manaka, and H. Nojiri, Phys. Rev. B 81, 100402(R) (2010).

[91] V. A. Stephanovich and M. E. Zhitomirsky, Europhys. Lett. 95, 17007 (2011).

[92] R. L. Doretto and M. Vojta, Phys. Rev. B 85, 104416 (2012).

[93] A. L. Chernyshev, Phys. Rev. B 86, 060401(R) (2012).

[94] W. T. Fuhrman, M. Mourigal, M. E. Zhitomirsky, and A. L. Chernyshev, Phys. Rev. B 85, 184405 (2012).

[95] M. E. Zhitomirsky and A. L. Chernyshev, Rev. Mod. Phys. 85, 219 (2013)

[96] J. Oh, M. D. Le, J. Jeong, J.-h. Lee, H. Woo, W.-Y. Song, T. G. Perring, W. J. L. Buyers, S.-W. Cheong, and J.-G. Park, Phys. Rev. Lett. 111, 257202 (2013).

[97] V. A. Stephanovich and M. E. Zhitomirsky, Phys. Rev. B 89, 224415 (2014).

[98] A. L. Chernyshev, Phys. Rev. B 92, 094409 (2015).

[99] A. L. Chernyshev and M. E. Zhitomirsky, Phys. Rev. B 92, 144415 (2015).

[100] Z. Z. Du, H. M. Liu, Y. L. Xie, Q. H. Wang, and J.-M. Liu, Phys. Rev. B 92, 214409 (2015).

[101] C. Luo, T. Datta, Z. Huang, and D.-X. Yao, Phys. Rev. B 92, 035109 (2015).

[102] J. Oh, M. D. Le, H.-H. Nahm, H. Sim, J. Jeong, T. G. Perring, H. Woo, K. Nakajima, S. Ohira-Kawamura, Z. Yamani, Y. Yoshida, H. Eisaki, S.-W. Cheong, A. L. Chernyshev, and J.-G. Park, Nat. Commun. 7, 13146 (2016).

[103] Z. Z. Du, H. M. Liu, Y. L. Xie, Q. H. Wang, and J.-M. Liu, Phys. Rev. B 94, 134416 (2016).

[104] A. L. Chernyshev and P. A. Maksimov, Phys. Rev. Lett. 117, 187203 (2016).
[105] S. M. Winter, K. Riedl, P. A. Maksimov, A. L. Chernyshev, A. Honecker, and R. Valentí, Nat. Commun. 8, 1152 (2017).

[106] T. Hong, Y. Qiu, M. Matsumoto, D. A. Tennant, K. Coester, K. P. Schmidt, F. F. Awwadi, M. M. Turnbull, H. Agrawal, and A. L. Chernyshev, Nat. Commun. 8, 15148 (2017).

[107] P. A. McClarty, X.-Y. Dong, M. Gohlke, J. G. Rau, F. Pollmann, R. Moessner, and K. Penc, Phys. Rev. B 98, 060404(R) (2018).

[108] L. Chaix, E. W. Huang, S. Gerber, X. Lu, C. Jia, Y. Huang, D. E. McNally, Y. Wang, F. H. Vernay, A. Keren, M. Shi, B. Moritz, Z.-X. Shen, T. Schmitt, T. P. Devereaux, and W.-S. Lee, Phys. Rev. B 97, 155144 (2018).

[109] P. A. McClarty and J. G. Rau, Phys. Rev. B 100, 100405(R) (2019).

[110] J. G. Rau, R. Moessner, and P. A. McClarty, Phys. Rev. B 100, 104423 (2019).

[111] T. Holstein and H. Primakoff, Phys. Rev. 58, 1098 (1940).

[112] I. Dzyaloshinsky, J. Phys. Chem. Solids 4, 241 (1958).

[113] T. Moriya, Phys. Rev. 120, 91 (1960).

[114] A. Fert and P. M. Levy, Phys. Rev. Lett. 44, 1538 (1980).

[115] A. Crépieux and C. Lacroix, J. Magn. Magn. Mater. 182, 341 (1998).

[116] J. H. Han, J. Zang, Z. Yang, J.-H. Park, and N. Nagaosa, Phys. Rev. B 82, 094429 (2010).

[117] S.-Z. Lin, C. D. Batista, and A. Saxena, Phys. Rev. B 89, 024415 (2014).

[118] O. Petrova and O. Tchernyshyov, Phys. Rev. B 84, 214433 (2011).

[119] Dynamics of Solids and Liquids by Neutron Scattering, edited by S. W. Lovesey and T. Springer (Springer, Berlin, 1977).

[120] Y. Onose, Y. Okamura, S. Seki, S. Ishiwata, and Y. Tokura, Phys. Rev. Lett. 109, 037603 (2012).

[121] Y. Okamura, F. Kagawa, M. Mochizuki, M. Kubota, S. Seki, S. Ishiwata, M. Kawasaki, Y. Onose, and Y. Tokura, Nat. Commun. 4, 2391 (2013).

[122] T. Schwarze, J. Waizner, M. Garst, A. Bauer, I. Stasinopoulos, H. Berger, C. Pfleiderer, and D. Grundler, Nat. Mater. 14, 478 (2015).

[123] D. Ehlers, I. Stasinopoulos, V. Tsurkan, H.-A. Krug von Nidda, T. Fehér, A. Leonov, I. Kézsmárki, D. Grundler, and A. Loidl, Phys. Rev. B 94, 014406 (2016).

[124] M. Ikka, A. Takeuchi, and M. Mochizuki, Phys. Rev. B 98, 184428 (2018).

[125] R. Chisnell, J. S. Helton, D. E. Freedman, D. K. Singh, R. I. Bewley, D. G. Nocera, and Y. S. Lee, Phys. Rev. Lett. 115, 147201 (2015).

[126] L. Chen, J.-H. Chung, B. Gao, T. Chen, M. B. Stone, A. I. Kolesnikov, Q. Huang, and P. Dai, Phys. Rev. X 8, 041028 (2018).

[127] H. Zhang, X. Feng, T. Heitmann, A. I. Kolesnikov, M. B. Stone, Y.-M. Lu, and X. Ke, Phys. Rev. B 101, 100405(R) (2020).

[128] S. Mizukami, D. Watanabe, M. Oogane, Y. Ando, Y. Miura, M. Shirai, and T. Miyazaki, J. Appl. Phys. 105, 07 D306 (2009).

[129] S. Mankovsky, D. Ködderitzsch, G. Woltersdorf, and H. Ebert, Phys. Rev. B 87, 014430 (2013).

[130] P. Dürrenfeld, F. Gerhard, J. Chico, R. K. Dumas, M. Ranjbar, A. Bergman, L. Bergqvist, A. Delin, C. Gould, L. W. Molenkamp, and J. Åkerman, Phys. Rev. B 92, 214424 (2015). 
[131] S. Andrieu, A. Neggache, T. Hauet, T. Devolder, A. Hallal, M. Chshiev, A. M. Bataille, P. Le Fèvre, and F. Bertran, Phys. Rev. B 93, 094417 (2016).

[132] M. A. W. Schoen, D. Thonig, M. L. Schneider, T. J. Silva, H. T. Nembach, O. Eriksson, O. Karis, and J. M. Shaw, Nat. Phys. 12, 839 (2016).

[133] S. Husain, S. Akansel, A. Kumar, P. Svedlindh, and S. Chaudhary, Sci. Rep. 6, 28692 (2016).

[134] A. J. Lee, J. T. Brangham, Y. Cheng, S. P. White, W. T. Ruane, B. D. Esser, D. W. McComb, P. C. Hammel, and F. Yang, Nat. Commun. 8, 234 (2017).

[135] Q. Qin, S. He, W. Song, P. Yang, Q. Wu, Y. P. Feng, and J. Chen, Appl. Phys. Lett. 110, 112401 (2017).

[136] J. M. Shaw, E. K. Delczeg-Czirjak, E. R. J. Edwards, Y. Kvashnin, D. Thonig, M. A. W. Schoen, M. Pufall, M. L. Schneider, T. J. Silva, O. Karis, K. P. Rice, O. Eriksson, and H. T. Nembach, Phys. Rev. B 97, 094420 (2018).

[137] L. Bainsla, R. Yilgin, M. Tsujikawa, K. Suzuki, M. Shirai, and S. Mizukami, J. Phys. D 51, 495001 (2018).

[138] I. Stasinopoulos, S. Weichselbaumer, A. Bauer, J. Waizner, H. Berger, S. Maendl, M. Garst, C. Pfleiderer, and D. Grundler, Appl. Phys. Lett. 111, 032408 (2017).

[139] M. Halder, A. Chacon, A. Bauer, W. Simeth, S. Mühlbauer, H. Berger, L. Heinen, M. Garst, A. Rosch, and C. Pfleiderer, Phys. Rev. B 98, 144429 (2018).

[140] Q. Tong, F. Liu, J. Xiao, and W. Yao, Nano Lett. 18, 7194 (2018).

[141] B. Ding, Z. Li, G. Xu, H. Li, Z. Hou, E. Liu, X. Xi, F. Xu, Y. Yao, and W. Wang, Nano Lett. 20, 868 (2019).

[142] A. K. Behera, S. Chowdhury, and S. R. Das, Appl. Phys. Lett. 114, 232402 (2019).

[143] J. Liang, W. Wang, H. Du, A. Hallal, K. Garcia, M. Chshiev, A. Fert, and H. Yang, Phys. Rev. B 101, 184401 (2020).

[144] J. Yuan, Y. Yang, Y. Cai, Y. Wu, Y. Chen, X. Yan, and L. Shen, Phys. Rev. B 101, 094420 (2020).
[145] T. Okubo, S. Chung, and H. Kawamura, Phys. Rev. Lett. 108, 017206 (2012).

[146] Y. Kamiya and C. D. Batista, Phys. Rev. X 4, 011023 (2014).

[147] A. O. Leonov and M. Mostovoy, Nat. Commun. 6, 8275 (2015).

[148] S. von Malottki, B. Dupé, P. F. Bessarab, A. Delin, and S. Heinze, Sci. Rep. 7, 12299 (2017).

[149] S. Hayami and Y. Motome, Phys. Rev. B 99, 094420 (2019).

[150] H. D. Rosales, D. C. Cabra, and P. Pujol, Phys. Rev. B 92, 214439 (2015).

[151] M. Li, N. B. Perkins, and I. Rousochatzakis, Phys. Rev. Research 1, 013002 (2019).

[152] S. Heinze, K. von Bergmann, M. Menzel, J. Brede, A. Kubetzka, R. Wiesendanger, G. Bihlmayer, and S. Blügel, Nat. Phys. 7, 713 (2011).

[153] R. F. Evans, W. J. Fan, P. Chureemart, T. A. Ostler, M. O. Ellis, and R. W. Chantrell, J. Phys.: Condens. Matter 26, 103202 (2014)

[154] J. Colpa, Physica A 93, 327 (1978).

[155] J. G. Rau, P. A. McClarty, and R. Moessner, Phys. Rev. Lett. 121, 237201 (2018).

[156] E. Rastelli, Statistical Mechanics of Magnetic Excitations (World Scientific, Singapore, 2011).

[157] R. N. Costa Filho, M. G. Cottam, and G. A. Farias, Phys. Rev. B 62, 6545 (2000).

[158] K. Schultheiss, R. Verba, F. Wehrmann, K. Wagner, L. Körber, T. Hula, T. Hache, A. Kákay, A. A. Awad, V. Tiberkevich, A. N. Slavin, J. Fassbender, and H. Schultheiss, Phys. Rev. Lett. 122, 097202 (2019).

[159] N. D. Mermin and H. Wagner, Phys. Rev. Lett. 17, 1133 (1966).

[160] F. Bloch, Z. Naturforsch. A 61, 206 (1930).

[161] S. Seki, M. Garst, J. Waizner, R. Takagi, N. D. Khanh, Y. Okamura, K. Kondou, F. Kagawa, Y. Otani, and Y. Tokura, Nat. Commun. 11, 256 (2020). 